\title{
Fidelity Lower Bounds for Stabilizer and CSS Quantum Codes
}

\author{
Alexei Ashikhmin, Senior Member, IEEE \\ Bell Laboratories, Alcatel-Lucent Inc. \\ Murray Hill, NJ 07974 \\ e-mail: aea@research.bell-labs.com
}

\begin{abstract}
In this paper we estimate the fidelity of stabilizer and CSS codes. First, we derive a lower bound on the fidelity of a stabilizer code via its quantum enumerator. Next, we find the average quantum enumerators of the ensembles of finite length stabilizer and CSS codes. We use the average quantum enumerators for obtaining lower bounds on the average fidelity of these ensembles. We further improve the fidelity bounds by estimating the quantum enumerators of expurgated ensembles of stabilizer and CSS codes. Finally, we derive fidelity bounds in the asymptotic regime when the code length tends to infinity.

These results tell us which code rate we can afford for achieving a target fidelity with codes of a given length. The results also show that in symmetric depolarizing channel a typical stabilizer code has better performance, in terms of fidelity and code rate, compared with a typical CSS codes, and that balanced CSS codes significantly outperform other CSS codes. Asymptotic results demonstrate that CSS codes have a fundamental performance loss compared to stabilizer codes.
\end{abstract}

\section{INTRODUCTION}

In recent years quantum error correcting codes were subject of intensive studies as they allow protection of quantum information from decoherence during quantum computations. The main focus of these studies was on various constructions of quantum codes, such as block codes, convolutional code, LDPC quantum codes and others, and their combinatorial, geometrical and topological properties, such as the minimum distance and others (see, for example, numerous publications in IEEE Trans. on Information Theory, Physical Review A, Physical Review Letters, International Journal of Quantum Information).

At the same time it is not so much known about the fidelity that one can hope to achieve using good quantum codes. A number of lower bounds on the fidelity $F$ of quantum codes in the asymptotic regime, as the code length tends to infinity, were derived in [9],[10],[3]. These bounds were derived in terms of reliability functions (error exponents).

In this article we consider the problem from a different angle. First, we would like to derive lower bounds on the fidelity $F$ as a function of the code length. While a reliability function tells us what kind of fidelity we may expect in the asymptotic regime, it does not give a good estimate on the fidelity for quantum codes of short and moderate code length, like 50 or 1000 qubits.

This work was supported by the Intelligence Advanced Research Projects Activity (IARPA) via Department of Interior National Business Center contract number D11PC20165.
Second, we would like to estimate the fidelity for several ensembles of quantum codes. In particular, we are interested in the ensembles of stabilizer codes, linear stabilizer codes, and CSS codes with different choices of parameters $k_{1}$ and $k_{2}$. CSS codes form an important subfamily of stabilizer codes. They are attractive for numerous applications, such as error correction in quantum memory, quantum fault-tolerant computations, quantum cryptography, and others. Therefore estimates on their fidelity can be very important for proper use of CSS codes in these applications.

Third, we are interested in analysis of performance of CSS codes in the asymptotic regime, as the code length tends to infinity. In particular, we would like to understand what is the fundamental performance loss of CSS codes compared with the performance of unrestricted stabilizer codes as the code length tends to infinity.

The paper is organized as follows. In Section I-A we remind the main definitions of quantum depolarizing channel. In Section II-B we remind the definitions of classical and quantum enumerators and their main properties, which will be used later in the paper. In Section $\amalg$ we derive a lower bound on the fidelity $F$ of quantum stabilizer code $Q$ as a function of its quantum enumerators. Further we derive two lower bounds on the average fidelity of an ensemble of quantum codes and apply these bounds to the ensemble of stabilizer codes of given length and code rate. In Section $\nabla$ we find the average quantum enumerators of the ensemble of CSS codes and apply them for obtaining lower bounds on the average fidelity of this ensemble. In Section VI we investigate the behavior of $F$ for stabilizer and CSS codes in the asymptotic regime as the code length tends to infinity.

\section{Preliminaries}

\section{A. Quantum Depolarizing Channel}

An $((n, K))$ binary quantum code $Q$ is a $K$-dimensional linear subspace of the complex space $\mathbb{C}^{2^{n}}$.

A quantum channel is a trace-preserving completely positive linear map $\mathcal{M}$. Any such map has an operator sum representation

$$
\mathcal{M}(\rho)=\sum_{k \in \mathcal{K}} M_{k} \rho M_{k}^{\dagger}
$$

for some operators $M_{k}$. Here $\rho$ is a density operator on $\mathbb{C}^{2^{n}}$ and $\mathcal{K}$ is a set of indices. We will write $\mathcal{M} \sim\left\{M_{k}\right\}_{k \in K}$. 
Decoding, or state-recovery operator, $\mathcal{R}$ associated with $Q$ is another trace-preserving completely positive linear map. The minimum fidelity of $Q$ is defined by

$$
F(Q, \mathcal{M}, \mathcal{R})=\min _{|\psi\rangle \in Q}\langle\psi|\mathcal{R} \mathcal{M}(|\psi\rangle\langle\psi|)| \psi\rangle .
$$

In what follows it will be more convenient for us to use the bound (8) (see Section III) instead of working with the definition (1) itself. More details on trace-preserving completely positive linear maps and fidelity of quantum codes can found in [9],[10], and references within.

The quantum symmetric depolarizing channel is defined with the help of Pauli operators

$$
\sigma_{x}=\left[\begin{array}{ll}
0 & 1 \\
1 & 0
\end{array}\right], \sigma_{z}=\left[\begin{array}{rr}
1 & 0 \\
0 & -1
\end{array}\right], \sigma_{y}=\left[\begin{array}{rr}
0 & i \\
-i & 0
\end{array}\right] .
$$

Denote by $0,1, \omega, \omega^{2}$ the elements of the Galois field $\mathbb{F}_{4}$. Let us associate with a vector $\mathbf{x}=\left(x_{1}, \ldots, x_{n}\right) \in \mathbb{F}_{4}^{n}$ the linear operator

$$
E_{\mathbf{x}}=e_{1} \otimes \ldots \otimes e_{n}
$$

where

$$
e_{j}= \begin{cases}I_{2}, & x_{j}=0, \\ \sigma_{x}, & x_{j}=1, \\ \sigma_{z}, & x_{j}=\omega, \\ \sigma_{y}, & x_{j}=\omega^{2} .\end{cases}
$$

The operators $E_{\mathbf{x}}$ are called error operators. The Hamming weight of $\mathrm{x}$ is defined in the standard way by

$$
\text { wt }(\mathbf{x})=\left|\left\{x_{j} \neq 0, j=1, \ldots, n\right\}\right| .
$$

The quantum symmetric depolarizing channel is the channel with the trace-preserving completely positive linear map

$$
\mathcal{M} \sim\left\{\left(\frac{p}{3}\right)^{\mathrm{wt}(\mathbf{x})}(1-p)^{n-\mathrm{wt}(\mathbf{x})} E_{\mathbf{x}}: \mathbf{x} \in \mathbb{F}_{4}^{n}\right\} .
$$

The quantity $p, 0 \leqslant p \leqslant 1$, is channel error probability.

Equivalently the quantum symmetric depolarizing channel can be defined as a channel in which the $j$-th qubit is effected by $e_{j} \in\left\{I_{2}, \sigma_{x}, \sigma_{y}, \sigma_{z}\right\}$ with probabilities

$$
\begin{gathered}
\operatorname{Pr}\left(e_{j}=I_{2}\right)=1-p, \\
\operatorname{Pr}\left(e_{j}=\sigma_{x}\right)=\operatorname{Pr}\left(e_{j}=\sigma_{z}\right)=\operatorname{Pr}\left(e_{j}=\sigma_{y}\right)=\frac{p}{3} .
\end{gathered}
$$

Thus a quantum code state $|\psi\rangle \in Q$ is effected by the error operator $E_{\mathbf{x}}$ with probability

$$
\operatorname{Pr}\left(E_{\mathbf{x}}\right)=\left(\frac{p}{3}\right)^{\mathrm{wt}(\mathbf{x})}(1-p)^{n-\mathrm{wt}(\mathbf{x})} .
$$

\section{B. Quantum Enumerators}

Important parameter of any classical linear code is its weight enumerator, or its weight distribution. The weight enumerator of a linear $[n, k]$ code $C$ over $\mathbb{F}_{q}$ is defined as the set of numbers:

$$
A_{j}(C)=|\{\mathbf{c} \in C: \operatorname{wt}(\mathbf{c})=j\}|, j=0, \ldots, n .
$$

The Euclidian dual code of $C$ is defined by

$$
\begin{aligned}
C^{\perp}= & \left\{\mathbf{c}=\left(c_{1}, \ldots, c_{n}\right): \mathbf{c} \cdot \mathbf{c}^{\prime}=0\right. \\
& \text { for all } \left.\mathbf{c}^{\prime}=\left(c_{1}^{\prime}, \ldots, c_{n}^{\prime}\right) \in C\right\},
\end{aligned}
$$

where

$$
\mathbf{c} \cdot \mathbf{c}^{\prime}=c_{1} c_{1}^{\prime}+\ldots+c_{n} c_{n}^{\prime}
$$

is the Euclidian inner product.

We say that a code $C$ over $\mathbb{F}_{4}$ is additive if $\mathbf{c}+\mathbf{c}^{\prime} \in C$ for any $\mathbf{c}, \mathbf{c}^{\prime} \in C$. The conjugate elements of $\mathbb{F}_{4}$ are defined by

$$
\overline{0}=0, \overline{1}=1, \bar{\omega}=\omega^{2}, \overline{\omega^{2}}=\omega .
$$

The Hermitian dual code of an additive code $C$ is defined by

$$
C^{\perp}=\left\{\mathbf{c}: \mathbf{c} * \mathbf{c}^{\prime}=0 \text { for all } \mathbf{c}^{\prime} \in C\right\},
$$

where

$$
\mathbf{c} * \mathbf{c}^{\prime}=\operatorname{Tr}_{\mathbb{F}_{2}}^{\mathbb{F}_{4}}\left(c_{1} \bar{c}_{1}^{\prime}+\ldots+c_{n} \bar{c}_{n}^{\prime}\right)
$$

is the trace inner product. Here $\operatorname{Tr}_{\mathbb{F}_{2}}^{\mathbb{F}_{4}}$ is the trace operator from $\mathbb{F}_{4}$ into $\mathbb{F}_{2}$. For codes over fields $\mathbb{F}_{q}, q>4$, their Hermitian dual codes are defined in [2].

In what follows we will use the same notation $C^{\perp}$ for both Euclidean and Hermitian dual codes. The meaning will be clear from the context.

If $C^{\perp}$ is Euclidean or Hermitian dual of $C$ over $\mathbb{F}_{q}$ then its weight enumerator is connected to the weight enumerator of $C$ via the MacWilliams identities:

$$
A_{j}^{\perp}=\frac{1}{|C|} \sum_{i=0}^{n} A_{i} \mathcal{K}_{j}(i), j=0, \ldots, n,
$$

where

$$
\mathcal{K}_{j}(i)=\sum_{t=0}^{j}(-q)^{t}(q-1)^{j-t}\left(\begin{array}{c}
n-t \\
j-t
\end{array}\right)\left(\begin{array}{l}
i \\
t
\end{array}\right)
$$

are Krawtchouk polynomials. Often it is more convenient to formulate the MacWilliams identities in the following polynomial form. Let

$$
A^{\perp}(x, y)=\sum_{j=0}^{n} A_{j}^{\perp} x^{n-j} y^{j} \text { and } A(x, y)=\sum_{j=0}^{n} A_{j} x^{n-j} y^{j} .
$$

Then

$$
A(x, y)=\frac{1}{\left|C^{\perp}\right|} A^{\perp}(x+(q-1) y, x-y) .
$$

In [16] P. Shor and R. Laflamme generalized the notion of weight enumerators for the case of quantum codes as follows. A quantum code $Q$ is a linear subspace of $\mathbb{C}^{2^{n}}$ and therefore there exists the orthogonal projector $P$ on $Q$. The code $Q$ has two quantum enumerators $B_{j}$ and $B_{j}^{\perp}$ defined by

$$
\begin{aligned}
B_{j}^{\perp}(Q) & =\frac{1}{\operatorname{dim}(Q)^{2}} \sum_{\mathbf{x} \in \mathbb{F}_{4}^{n}: \operatorname{wt}(\mathbf{x})=j} \operatorname{Tr}\left(E_{\mathbf{x}} P\right)^{2}, j=0, \ldots, n, \\
B_{j}(Q) & =\frac{1}{\operatorname{dim}(Q)} \sum_{\mathbf{x} \in \mathbb{F}_{4}^{n}: \operatorname{wt}(\mathbf{x})=j} \operatorname{Tr}\left(E_{\mathbf{x}} P E_{\mathbf{x}} P\right), j=0, \ldots, n .
\end{aligned}
$$

In this paper we are interested only in quantum binary stabilizer codes. A binary $[[n, k]]$ quantum stabilizer code $Q$ is a $2^{k}$-dimensional linear subspace of $\mathbb{C}^{2^{n}}$ that is defined by a classical additive code $C$ of length $n$ and size $2^{n+k}$ over $\mathbb{F}_{4}$. The code $C$ has the property that its Hermitian dual $C^{\perp}$ is a subset of $C$, that is $C^{\perp} \subseteq C$. See [8],[18],[5] for the exact definition of stabilizer codes. If $C$ is a linear code over 
$\mathbb{F}_{4}$ then the corresponding quantum code $Q$ is called linear stabilizer code.

Denote by

$$
R\left(C^{\perp}\right)=\frac{1}{n} \log _{4}\left|C^{\perp}\right| \text { and } R(C)=\frac{1}{n} \log _{4}|C|
$$

the code rates of $C^{\perp}$ and $C$ respectively. These codes rates are connected to the code rate $R=\frac{k}{n}$ of $Q$ by

$$
R\left(C^{\perp}\right)=\frac{1-R}{2} \text { and } R(C)=\frac{R+1}{2} .
$$

The quantum enumerators of a stabilizer code $Q$ are equal to the enumerators of $C$ and $C^{\perp}$, that is

$$
B_{j}(Q)=A_{j}(C), B_{j}^{\perp}(Q)=A_{j}\left(C^{\perp}\right) .
$$

The quantum enumerators of a stabilizer code have a number of useful properties. In particular,

1) The enumerators $B_{j}$ and $B_{j}^{\perp}$ are nonnegative integers and

$$
B_{j} \geqslant B_{j}^{\perp}, B_{0}^{\perp}=B_{0}=1 .
$$

2) If $t$ is the smallest integer such that $B_{t}>B_{t}^{\perp}$ then the minimum distance of $Q$ is $d(Q)=t$.

3) The sum of $B_{j}$ defines the size of $Q$ :

$$
\operatorname{dim}(Q)=2^{k}=\frac{1}{2^{n}} \sum_{j=0}^{n} B_{j}=2^{n} \cdot \frac{1}{\sum_{j=0}^{n} B_{j}^{\perp}} .
$$

4) Similar to the classical case quantum enumerators are connected to each other via the MacWilliams identities

$$
B_{r}=\frac{\operatorname{dim}(Q)}{2^{n}} \sum_{j=0}^{n} B_{j}^{\perp} \mathcal{K}_{r}(j),
$$

where $\mathcal{K}_{r}(j)$ is the quaternary Krawtchouk polynomial defined in (3). In the polynomial form the quantum MacWilliams identities have the form

$$
B(x, y)=\frac{\operatorname{dim}(Q)}{2^{n}} B^{\perp}(x+3 y, x-y),
$$

where

$$
\begin{aligned}
B^{\perp}(x, y) & =\sum_{j=0}^{n} B_{j}^{\perp} x^{n-j} y^{j}, \text { and } \\
B(x, y) & =\sum_{j=0}^{n} B_{j} x^{n-j} y^{j} .
\end{aligned}
$$

\section{BOUND ON Fidelity VIA QUANTUM ENUMERATORS}

Correctable and uncorrectable errors of a classical linear code in $q$-ary symmetric channel can be characterized with the help of its standard array, see for example [4, Ch.3.3]. For a quantum stabilizer code $Q$ associated with classical code $C$ one can generalize the standard array as it is shown in Fig 1

Remind that a coset of a linear code $C$ generated by a vector $\mathbf{v}$ is the set

$$
C+\mathbf{v}=\{\mathbf{c}+\mathbf{v}: \mathbf{c} \in C\} .
$$

The space $\mathbb{F}_{4}^{n}$ can be partitioned into the cosets of $C$. Each coset of $C$, say $C+\mathbf{v}_{l}, \mathbf{v}_{l} \in \mathbb{F}_{4}^{n}$, can be further partitioned into cosets of $C^{\perp}$ for appropriately chosen coset leaders $\mathbf{a}_{0}, \ldots, \mathbf{a}_{2^{2 k}-1} \in C \backslash C^{\perp}$. The cosets of $C^{\perp}$ can be permuted inside the coset $C+\mathbf{v}_{l}$. For example, we can assign $\mathbf{v}_{l}^{\prime}=$ $\mathbf{v}_{l}+\mathbf{a}_{j}$, and use $\mathbf{v}_{l}^{\prime}$ instead of $\mathbf{v}_{l}$. After this permutation the leading (the most left) coset of $C^{\perp}$ in the $l$-th row of the array will be $C^{\perp}+\mathbf{v}_{l}+\mathbf{a}_{j}$.

Any vector $\mathrm{x} \in \mathbb{F}_{4}^{n}$ appears in the standard array one and only one time. The error operators $E_{\mathbf{x}}$ that correspond to the vectors $\mathbf{x}$ from the leading (the most left) cosets $C^{\perp}+\mathbf{v}_{l}, l=$ $0, \ldots, 2^{n-k}-1$ (here we assume $\mathbf{v}_{0}=(0, \ldots, 0)$ ) form the set $J$ of correctable error operators (see [8],[5] and references within).

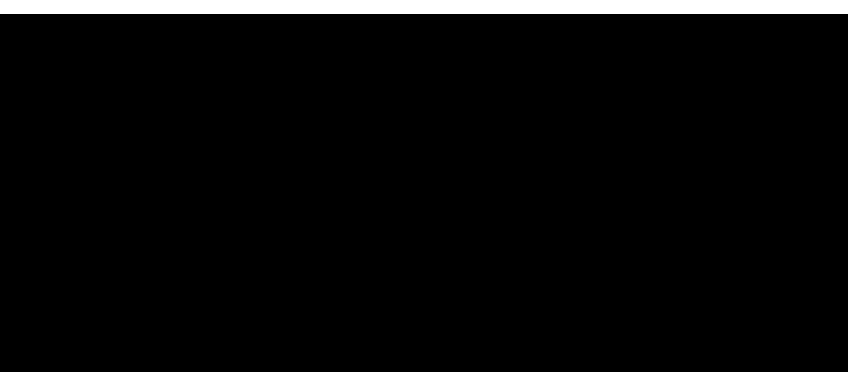

Fig. 1. Standard Array for Stabilizer Codes. Here $M=2^{2 k}-1$ and $N=2^{n-k}-1$.

In [9],[10] M. Hamada proved, using a result from [15], the following lower bound on minimum average fidelity of $Q$ :

$$
F(Q) \geqslant 1-\sum_{\mathbf{x} \notin J} \operatorname{Pr}\left(E_{\mathbf{x}}\right) .
$$

As we discussed above any coset $C^{\perp}+\mathbf{v}_{l}+\mathbf{a}_{j}, j=$ $0, \ldots, 2^{2 k}-1$, (here $\mathbf{a}_{0}=(0, \ldots, 0)$ ) can be used as the leading coset in the $l$-th row of the standard array. The optimal choice $j^{*}$, which maximizes $F(Q)$, is defined by

$$
j^{*}=\arg \max _{0 \leqslant j \leqslant 2^{2 k}-1} \sum_{\mathbf{c} \in C^{\perp}} \operatorname{Pr}\left(E_{\mathbf{c}+\mathbf{v}_{l}+\mathbf{a}_{j}}\right) .
$$

The value $j^{*}$ is not fixed and depends on the channel error probability $p$. So for different values of $p$ we may have different $j^{*}$ s. For deriving a lower bound on $F(Q)$ we may choose any coset $C^{\perp}+\mathbf{v}_{l}+\mathbf{a}_{j}$ as the leading one in the $l$-th row of the standard array. Our goal, of course, is to choose it such that to get large $F(Q)$. We will use the "classical" approach. Let $\mathbf{w} \in C+\mathbf{v}_{l}$ be a minimum weight vector in the $l$-th row of the standard array, that is

$$
\mathrm{wt}(\mathbf{w}) \leqslant \mathrm{wt}(\mathbf{v}) \text { for all } \mathbf{v} \in C+\mathbf{v}_{l} \text {. }
$$

Then we choose as a leading coset the one that contains $\mathbf{w}$, that is we chose $j$ such that

$$
\mathbf{w} \in C^{\perp}+\mathbf{v}_{l}+\mathbf{a}_{j} .
$$

Examples show that when $p$ is not too large this is a good and, in fact, most likely the optimal choice of the leading coset.

In [13] G. Poltyrev derived an upper bound on the probability of decoding error of classical linear code via its weight enumerator $A_{j}$. The following theorem generalizes this bound for quantum stabilizer codes. 
Theorem 1: Let $Q$ be a stabilizer code of length $n$ with quantum enumerators $B_{j}^{\perp}$ and $B_{j}$. Then

$$
\begin{aligned}
& 1-F(Q) \\
\leqslant & \sum_{m=1}^{n}\left(\frac{p}{3}\right)^{m}(1-p)^{n-m} \min \left\{\left(\begin{array}{c}
n \\
m
\end{array}\right) 3^{m}, N(m)\right\},
\end{aligned}
$$

where

$$
N(m)=\sum_{w=1}^{\min (2 m, n)}\left(B_{w}-B_{w}^{\perp}\right) G(m, w),
$$

and

$$
\begin{aligned}
& G(m, w) \\
= & \sum_{t=0}^{m}\left(\begin{array}{c}
w \\
t
\end{array}\right) 2^{t} \sum_{h=\left\lceil\frac{w-t}{2}\right\rceil}^{m-t}\left(\begin{array}{c}
w-t \\
h
\end{array}\right)\left(\begin{array}{c}
n-w \\
m-t-h
\end{array}\right) 3^{m-t-h}
\end{aligned}
$$

Proof: Let us consider an error vector

$$
\mathbf{x}=\left(x_{1}, \ldots, x_{n}\right), \text { wt }(\mathbf{x})=m .
$$

According to our choice of the leading cosets in the standard array, the vector $\mathbf{x}$ may belong to $\mathbb{F}_{4}^{n} \backslash J$ only if $d(\mathbf{x}, \mathbf{c}) \leqslant$ $d(\mathbf{x}, \mathbf{0})$ for some $\mathbf{c}=\left(c_{1}, \ldots, c_{n}\right) \in C \backslash C^{\perp}$. Let $\mathbf{w t}(\mathbf{c})=w$ and denote

$$
h=\left|\left\{i: x_{i} \neq 0, x_{i}=c_{i}\right\}\right| \text { and } t=\left|\left\{i: x_{i} \neq 0, x_{i} \neq c_{i}\right\}\right| .
$$

Then

$$
m-h-t=\left|\left\{i: x_{i} \neq 0, c_{i}=0\right\}\right| .
$$

Using the above notations we get

$$
\begin{aligned}
& d(\mathbf{x}, \mathbf{c})=w-2 h-t+m, \\
& d(\mathbf{x}, \mathbf{0})=m .
\end{aligned}
$$

Using (12) and (13) we get that the number of vectors $\mathbf{x}$ for which $d(\mathbf{x}, \mathbf{c}) \leqslant d(\mathbf{x}, \mathbf{0})=m$ is equal to $G(m, w)$.

Using union bound we can upper bound the number of error vectors $\mathbf{x} \in \mathbb{F}_{4}^{n} \backslash J$, wt $(\mathbf{x})=m$, by $N(m)$. Taking into account that the total number of error vectors $\mathbf{x}, \mathbf{w t}(\mathbf{x})=m$, is $\left(\begin{array}{c}n \\ m\end{array}\right) 3^{m}$ we obtain (9).

\section{Bounds on the AVERAge Fidelity OF AN ENSEMBLE OF QuANTUM CODES}

In this section we derive bounds on the average fidelity over the ensemble of stabilizer codes. These bounds can be considered as achievability bounds in the sense that they prove existence of codes whose fidelity is at least as good as the bounds.

\section{A. Bound on the Average Fidelity}

Denote by $\mathcal{Q}_{\text {stab }}$ the ensemble of all $[[n, k]]$ stabilizer codes, that is

$$
\mathcal{Q}_{\text {stab }}=\{Q: Q \text { is an }[[n, k]] \text { code }\} \text {. }
$$

Let $\mathcal{Q}$ be an arbitrary sub-ensemble of $\mathcal{Q}_{\text {stab }}$. For instance $\mathcal{Q}$ can be $\mathcal{Q}_{s t a b}$ itself, or it can be the ensemble of all $\left(n, k_{1}, k_{2}\right)$
CSS codes (see Section $\mathrm{V}$ ). The average enumerators of $\mathcal{Q}$ are defined as

$$
\bar{B}_{j}^{\perp}=\frac{1}{|\mathcal{Q}|} \sum_{Q \in \mathcal{Q}} B_{j}^{\perp}(Q), \bar{B}_{j}=\frac{1}{|\mathcal{Q}|} \sum_{Q \in \mathcal{Q}} B_{j}(Q) .
$$

Similar to the classical case [14][Theorem 5], using the average enumerators $\bar{B}_{j}^{\perp}$ and $\bar{B}_{j}$ in Theorem 1 , we can obtain an upper bound on the average value of $1-F$ over the ensemble $\mathcal{Q}$. We formulate it as a Corollary of Theorem 1

\section{Corollary 2:}

$$
\begin{aligned}
& 1-\bar{F}=\frac{1}{|\mathcal{Q}|} \sum_{Q \in \mathcal{Q}}(1-F(Q)) \\
& \leqslant \sum_{m=d_{\text {min }} / 2}^{n}\left(\frac{p}{3}\right)^{m}(1-p)^{n-m} \min \left\{\left(\begin{array}{c}
n \\
m
\end{array}\right) 3^{m}, \bar{N}(m)\right\},
\end{aligned}
$$

where

$$
\bar{N}(m)=\sum_{w=d_{\min }}^{\min (2 m, n)}\left(\bar{B}_{w}-\bar{B}_{w}^{\perp}\right) G(m, w),
$$

Proof: The proof is identical to the classical case. The claim immediately follows from the observation that the function $\min \{\cdot, \cdot\}$ is $\bigcap$-convex and therefore Jensen's inequality can be applied to 9 .

Corollary 2 is an achievability bound in the sense that it guarantees that in $\mathcal{Q}$ there exists at least one code $Q$ with $F(Q) \geqslant \bar{F}$. Thus studying the average enumerators of an ensemble of stabilizer codes we get an insight about the fidelity of the codes from this ensemble.

Denote by $\mathcal{Q}_{\text {lin.stab }}$ the ensemble of all $[[n, k]]$ linear stabilizer codes, that is

$$
\mathcal{Q}_{\text {lin.stab }}=\{Q: Q \text { is a linear }[[n, k]] \text { code }\} \text {. }
$$

The average quantum enumerators of codes from the ensemble $\mathcal{Q}_{\text {lin.stab }}$ were found in [1]:

$$
\begin{aligned}
\bar{B}_{0}^{\perp} & =1, \\
\bar{B}_{j}^{\perp} & =\left(\begin{array}{c}
n \\
j
\end{array}\right) \frac{\alpha}{2}\left(3^{j}+(-3)^{j}\right), j \geqslant 1, \\
\bar{B}_{0} & =1 \\
\bar{B}_{j} & =\frac{1}{4^{(n-k) / 2}}\left(\begin{array}{c}
n \\
j
\end{array}\right)\left((1-\alpha) 3^{j}+\frac{\alpha}{2} 6^{j}(-2)^{n-j}\right), j \geqslant 1,
\end{aligned}
$$

where

$$
\alpha=\frac{4^{(n-k) / 2}-1}{\frac{1}{2}\left(4^{n}+(-2)^{n}\right)-1} .
$$

Below we find the average enumerators for the ensemble $\mathcal{Q}_{\text {stab. }}$. Let

$$
\mathcal{S}_{n, t}=\left\{C^{\perp} \subset \mathbb{F}_{4}^{n}:\left|C^{\perp}\right|=2^{t} \text { and } C^{\perp} \subset C\right\}
$$

be the ensemble of self-orthogonal codes of length $n$ and size $2^{t}$. We need with the following result. 
Lemma 3: 1. The number of codes in $\mathcal{S}_{n, t}$ is

$$
S(n, t)=\left|\mathcal{S}_{n, t}\right|=\prod_{r=0}^{t-1} \frac{2^{2(n-r)}-1}{2^{r+1}-1} .
$$

2. Any vector $\mathbf{v} \in \mathbb{F}_{4}^{n} \backslash \mathbf{0}$ is contained in

$$
L(n, t)=\prod_{r=1}^{t-1} \frac{2^{2(n-r)}-1}{2^{r}-1}
$$

codes from $\mathcal{S}_{n, t}$.

Proof: Let $C_{r}^{\perp} \in \mathcal{S}_{n, r}$ and $C_{r}$ be the dual of $C_{r}^{\perp}$. Any vector $\mathbf{w} \in \mathbb{F}_{4}^{n}$ is self-orthogonal with respect to the trace inner product (2), i.e. $\mathbf{w} * \mathbf{w}=0$. Hence if we take any $\mathbf{w} \in$ $C_{r} \backslash C_{r}^{\perp}$ then the code $C_{r+1}^{\perp}=C_{r}^{\perp} \bigcup\left(C_{r}^{\perp}+\mathbf{w}\right)$ is again selforthogonal. Using instead of $\mathbf{w}$ any other vector from the coset $\left(C_{r}^{\perp}+\mathbf{w}\right)$ we obtain the same code $C_{r+1}^{\perp}$. Since $\left|C_{r} \backslash C_{r}^{\perp}\right|=$ $2^{2 n-r}-2^{r}$ we can construct $\left(2^{2 n-r}-2^{r}\right) / 2^{r}$ different codes $C_{r+1}^{\perp}$ from a given $C_{r}^{\perp}$. In $C_{r+1}^{\perp}$ there exist $2^{r+1}-1$ different subcodes of size $2^{r}$. Hence

$$
S(n, r+1)=\frac{2^{2 n-r}-2^{r}}{2^{r}\left(2^{r+1}-1\right)} S(n, r) .
$$

From this expression (19) follows.

Let $C_{1}^{\perp}=\{\mathbf{0}, \mathbf{v}\}$. This code is self-orthogonal and can be used as a starting point for construction of codes $C_{r+1}^{\perp}$ in the above procedure. The only difference from the above procedure is that in $C_{r+1}^{\perp}$ there exist $2^{r}-1$ codes $C_{r}^{\perp}$ such that $C_{1}^{\perp} \subset C_{r}^{\perp}$ (see Lemma 11). Taking this into account we obtain the expression (20).

Now we can find the average enumerators of stabilizer codes.

Theorem 4: For the ensemble $\mathcal{Q}_{\text {stab }}$ we have

$$
\begin{gathered}
\bar{B}_{0}^{\perp}=1, \bar{B}_{j}^{\perp}=\frac{2^{n-k}-1}{4^{n}-1}\left(\begin{array}{l}
n \\
j
\end{array}\right) 3^{j}, j \geqslant 1, \\
\bar{B}_{0}=1, \bar{B}_{j}=\frac{2^{n+k}-1}{4^{n}-1}\left(\begin{array}{l}
n \\
j
\end{array}\right) 3^{j}, j \geqslant 1 .
\end{gathered}
$$

Proof: Because of (5), the enumerator $\bar{B}_{j}^{\perp}$ coincides with the average enumerator of classical codes from the ensemble $\mathcal{S}_{n, n-k}$, which is easy to find. Indeed, the number of vectors $\mathbf{w} \in \mathbb{F}_{4}^{n}$ of weight $j$ is $\left(\begin{array}{c}n \\ j\end{array}\right) 3^{j}$. From Lemma 3 we have

$$
\frac{L(n, n-k)}{S(n, n-k)}=\frac{2^{n-k}-1}{4^{n}-1}
$$

and the expression (21) follows.

Let us denote $\gamma=\frac{2^{n-k}-1}{4^{n}-1}$. It is easy to see that

$$
\bar{B}^{\perp}(x, y)=\sum_{j=0}^{n} \bar{B}_{j}^{\perp} y^{j} x^{n-j}=(1-\gamma) x^{n}+\gamma(x+3 y)^{n}
$$

Using the quantum MacWilliams identities (7), we obtain

$$
\begin{aligned}
\bar{B}(x, y) & =\sum_{j=0}^{n} \bar{B}_{j} y^{j} x^{n-j} \\
& =2^{k-n}\left((1-\gamma)(x+3 y)^{n}+\gamma(4 x)^{n}\right) .
\end{aligned}
$$

From this the expression (22) follows.

Using (21) and (22) in (14) we obtain a bound on $1-\bar{F}$ for the ensemble $\mathcal{Q}_{\text {stab. }}$. This bound for $n=50$ and $k=22$ is presented in Fig. 3 .

\section{B. Bound on the Average Fidelity of Expurgated Ensemble}

Below we show that for small values of the channel error $p$ one can significantly improve the bound (14). In order of doing this we first note that after simple algebraic manipulations the bound (9) can be transformed to the following form.

Let $m_{0}$ be the smallest integer such that

$$
N\left(m_{0}\right) \geqslant\left(\begin{array}{c}
n \\
m_{0}
\end{array}\right) 3^{m_{0}} .
$$

and let

$$
\begin{aligned}
T_{w} & =\left(B_{w}-B_{w}^{\perp}\right) \sum_{t=0}^{m_{0}-1}\left(\begin{array}{c}
w \\
t
\end{array}\right) 2^{t} \sum_{h=\left\lceil\frac{w-t}{2}\right\rceil}^{m_{0}-t-1}\left(\begin{array}{c}
w-t \\
h
\end{array}\right) \\
& \times \sum_{\kappa=0}^{m_{0}-1-t-h}\left(\begin{array}{c}
n-w \\
\kappa
\end{array}\right) 3^{\kappa}(p / 3)^{\kappa+t+h}(1-p)^{n-\kappa-t-h}
\end{aligned}
$$

Then

$$
1-F \leqslant \sum_{w=1}^{2\left(m_{0}-1\right)} T_{w}+\sum_{l=m_{0}}^{n}\left(\begin{array}{l}
n \\
l
\end{array}\right)(p / 3)^{l}(1-p)^{n-l} .
$$

Define $\bar{T}_{w}$ as in (24), but replacing $B_{j}$ and $B_{j}$ with $\bar{B}_{j}$ and $\bar{B}_{j}$ respectively. The right hand side of (25) is equal to the right hand side of (9). Hence if we use $\bar{N}(m)$ (defined in (15)) in (23) and $\bar{T}_{w}$ in (25) we obtain

$$
1-\bar{F} \leqslant \sum_{w=d_{m i n}}^{2\left(m_{0}-1\right)} \bar{T}_{w}+\sum_{l=m_{0}}^{n}\left(\begin{array}{l}
n \\
l
\end{array}\right)(p / 3)^{l}(1-p)^{n-l} .
$$

Let us now examine the individual contributions of the terms of (26). For instance, in the case of the ensemble of all $[[50,22]]$ stabilizer codes and $p=10^{-4}$ the values of $\bar{T}_{w}$ are shown in Fig.2. One can see that the main contributions are made by $\bar{T}_{2}$ and $\bar{T}_{4}$.

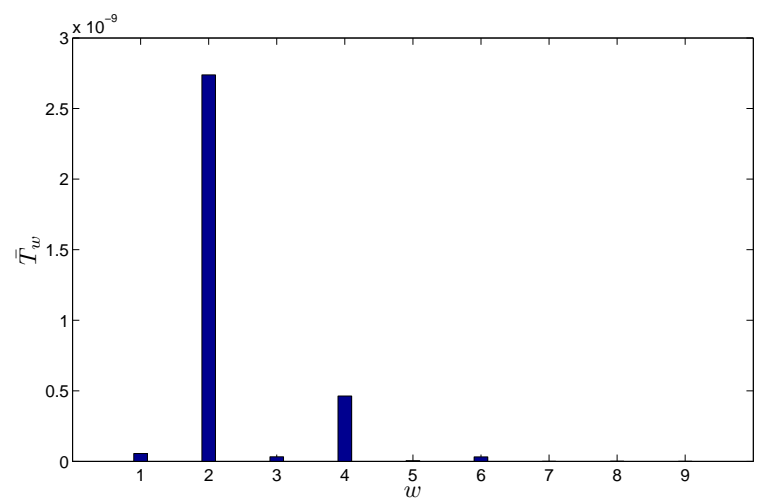

Fig. 2. $\quad \bar{T}_{w}$ for the ensemble of all $[[50,22]]$ stabilizer codes

In [7] the following lower bound on the minimum distance of stabilizer (pure) codes was proven.

Theorem 5: [7] For $n>k \geqslant 2, d \geqslant 2$ and $n=k \bmod 2$ there exists a pure stabilizer code $\left[\left[n, k, d_{G V}(n, k)\right]\right]$ provided

$$
\frac{2^{n-k+2}-1}{3}>\sum_{i=1}^{d_{G V}(n, k)-1} 3^{i-1}\left(\begin{array}{l}
n \\
i
\end{array}\right) \text {. }
$$


(For $n \neq k \bmod 2$ a similar bound can be derived.) According to this bound there exist $[[50,22]]$ codes with $d \geqslant 5$. For such codes, using the property 2 of $B_{j}$ and $B_{j}^{\perp}$ from Section II-B we get

$$
\bar{T}_{1}=\bar{T}_{2}=\bar{T}_{3}=\bar{T}_{4}=0 .
$$

So, one can hope that codes with $d \geqslant 5$ will have significantly smaller $1-\bar{F}$ than an average $[[50,22]]$ code.

Let $\mathcal{Q}$ be a sub-ensemble of $\mathcal{Q}_{s t a b}$ and let $\bar{B}_{j}^{\perp}$ and $\bar{B}_{j}$ be the average enumerators of $\mathcal{Q}$. Assume that there is at least one $j$ such that $\bar{B}_{j}-\bar{B}_{j}^{\perp}<1$ and consider a set

$$
I \subseteq\left\{j: \bar{B}_{j}-\bar{B}_{j}^{\perp}<1\right\} .
$$

Define the expurgated ensemble of $\mathcal{Q}_{e x}$ as

$$
\mathcal{Q}_{e x}=\left\{Q \in \mathcal{Q}: B_{j}(Q)-B_{j}^{\perp}(Q)=0 \text { for all } j \in I\right\} .
$$

For upper-bounding the average value of $1-F$ over $\mathcal{Q}_{e x}$, we have to estimate the average quantum enumerators of $\mathcal{Q}_{\text {ex }}$. The following theorem gives an upper bound on the average quantum enumerators of $\mathcal{Q}_{e x}$ in terms of the average enumerators of the original ensemble $\mathcal{Q}$.

Theorem 6: Let $\hat{B}_{j}^{\perp}$ and $\hat{B}_{j}$ be the average quantum enumerators of $\mathcal{Q}_{e x}$ and let

$$
\beta=1-\sum_{j \in I}\left(\bar{B}_{j}-\bar{B}_{j}^{\perp}\right) .
$$

If $\beta>0$ then $\mathcal{Q}_{e x}$ is not empty and

$$
\hat{B}_{j}-\hat{B}_{j}^{\perp} \leqslant \frac{1}{\beta}\left(\bar{B}_{j}-\bar{B}_{j}^{\perp}\right), j \notin I .
$$

Proof: Let $Q$ be a randomly chosen code from $\mathcal{Q}$ with respect to the uniform distribution. Then from Markov's inequality it follows that for a positive $\alpha_{j}$ we have

$$
\operatorname{Pr}\left(\left(B_{j}(Q)-B_{j}^{\perp}(Q)\right) \geqslant \alpha_{j}\left(\bar{B}_{j}-\bar{B}_{j}^{\perp}\right)\right) \leqslant \frac{1}{\alpha_{j}} .
$$

For $I=\left\{j_{1}, \ldots, j_{m}\right\} \subseteq\left\{j: \bar{B}_{j}-\bar{B}_{j}^{\perp}<1\right\}$, using the union bound, we obtain

$$
\begin{aligned}
& \operatorname{Pr}\left(B_{j_{r}}(Q)-B_{j_{r}}^{\perp}(Q) \leqslant \alpha_{j_{r}}\left(\bar{B}_{j_{r}}-\bar{B}_{j_{r}}^{\perp}\right) \text { for all } j_{r}\right) \\
\geqslant & 1-\frac{1}{\alpha_{j_{1}}}-\ldots-\frac{1}{\alpha_{j_{m}}} .
\end{aligned}
$$

Let

$$
\alpha_{j_{r}}=\frac{1-\epsilon}{\bar{B}_{j_{r}}-\bar{B}_{j_{r}}^{\perp}}, \epsilon>0 .
$$

If $\beta>0$ then we can choose $\epsilon>0$ such that the right hand side of 27) is positive. Hence there exists $Q$ such that

$$
B_{j_{r}}(Q)-B_{j_{r}}^{\perp}(Q) \leqslant \alpha_{j_{r}}\left(\bar{B}_{j}-\bar{B}_{j}^{\perp}\right), \text { for all } j_{r} \in I \text {. }
$$

Since $\alpha_{j_{r}}\left(\bar{B}_{j_{r}}-\bar{B}_{j_{r}}^{\perp}\right)<1$ and $B_{j}(Q)-B_{j}^{\perp}(Q)$ are integers (according to the first property of quantum enumerators in Section [I-B), we have

$$
B_{j_{r}}(Q)-B_{j_{r}}^{\perp}(Q)=0, \text { for all } j_{r} \in I .
$$

Summarizing this, we conclude that there exists a nonempty ensemble

$$
\mathcal{Q}_{e x} \subseteq \mathcal{Q}
$$

of stabilizer codes and for $Q \in \mathcal{Q}_{e x}$ we have $B_{j}(Q)-$ $B_{j}^{\perp}(Q)=0, j \in I$.

From (27) we have $\left|\mathcal{Q}_{e x}\right| \geqslant\lceil\beta|\mathcal{Q}|\rceil$. Hence we can get the following upper bound on $\hat{B}_{j}-\hat{B}_{j}^{\perp}$ :

$$
\begin{aligned}
& \bar{B}_{j}-\bar{B}_{j}^{\perp} \\
= & \frac{1}{|\mathcal{Q}|}\left(\sum_{Q \in \mathcal{Q}_{e x}}\left(B_{j}(Q)-B_{j}^{\perp}(Q)\right)+\sum_{Q \notin \mathcal{Q}_{e x}}\left(B_{j}(Q)-B_{j}^{\perp}(Q)\right)\right) \\
\geqslant & \frac{1}{|\mathcal{Q}|} \sum_{Q \in \mathcal{Q}_{e x}}\left(B_{j}(Q)-B_{j}^{\perp}(Q)\right) \\
= & \frac{|\mathcal{Q} e x|}{|\mathcal{Q}|}\left(\hat{B}_{j}-\hat{B}_{j}^{\perp}\right) \geqslant \beta\left(\hat{B}_{j}-\hat{B}_{j}^{\perp}\right) .
\end{aligned}
$$

Example 7: Let $\mathcal{Q}$ be the ensemble of all [[50,22]] stabilizer codes. Then we have

$$
\begin{aligned}
\bar{B}_{1}-\bar{B}_{1}^{\perp} \approx 5.6 \cdot 10^{-5}, & \bar{B}_{2}-\bar{B}_{2}^{\perp} \approx 4 \cdot 10^{-5}, \\
\bar{B}_{3}-\bar{B}_{3}^{\perp} \approx 2 \cdot 10^{-3}, & \bar{B}_{4}-\bar{B}_{4}^{\perp} \approx 6.9 \cdot 10^{-2} .
\end{aligned}
$$

Let us form $\mathcal{Q}_{e x}$ with $I=\{1,2,3,4\}$. In this case we have $\beta>0.9285$. Thus for the expurgated ensemble $\mathcal{Q}_{\text {ex }}$ we have

$$
\begin{array}{ll}
\hat{B}_{1}-\hat{B}_{1}^{\perp}=0, & \hat{B}_{2}-\hat{B}_{2}^{\perp}=0, \\
\hat{B}_{3}-\hat{B}_{3}^{\perp}=0, & \hat{B}_{4}-\hat{B}_{4}^{\perp}=0 .
\end{array}
$$

and for $j>4$

$$
\left(\hat{B}_{j}-\hat{B}_{j}^{\perp}\right) \leqslant 1.077 \cdot\left(\bar{B}_{j}-\bar{B}_{j}^{\perp}\right) .
$$

Upper bounds on $1-\bar{F}$ for $\mathcal{Q}$ and $\mathcal{Q}_{\text {ex }}$ are shown in fig 3 .

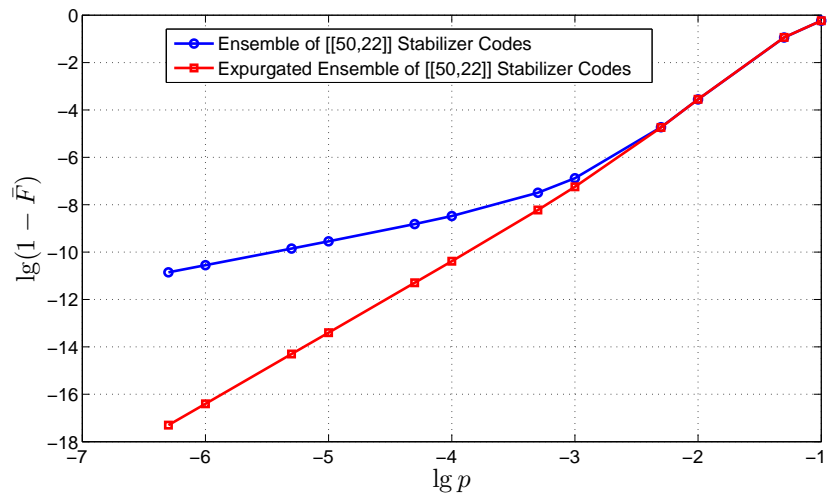

Fig. 3. Lower bounds on $1-\bar{F}$ for $\mathcal{Q}_{s t a b}$ and $\mathcal{Q}_{\text {stab,ex }}$

One can see that when $p<10^{-3}$ the bound for the expurgated ensemble $\mathcal{Q}_{e x}$ is getting significantly better than the bound for $\mathcal{Q}_{s t a b}$.

Remark Typically the average enumerators (21) and 22) of stabilizer codes are very close to the average enumerators 17 and (18) of linear stabilizer codes. This results in that the fidelity bounds for these ensembles are also basically identical. All results presented for stabilizer codes in Fig 3 , 4 , and 5 , and are also valid for linear stabilizer codes with the same parameters. 


\section{BOUNDS ON THE FIDELITY OF CSS CODES}

CSS codes [6], [17] form an important subclass of stabilizer codes. CSS codes are good candidates for some practical applications, such as quantum cryptographical protocols and fault-tolerant quantum computations. Hence it looks natural to try to estimate their performance and compare it with the performance of unrestricted stabilizer codes.

CSS codes form a subfamily of stabilizer codes. It is more convenient to define them with the help of binary classical codes, rather than classical codes over $\mathbb{F}_{4}$.

Remind that any vector $\mathbf{v} \in \mathbb{F}_{4}^{n}$ can be written in the form $\mathbf{v}=\mathbf{a}+\omega \mathbf{b}$, where $\mathbf{a}=\left(a_{1}, \ldots, a_{n}\right), \mathbf{b}=\left(b_{1}, \ldots, b_{n}\right) \in \mathbb{F}_{2}^{n}$. Note that

$$
\operatorname{wt}(\mathbf{v})=\mid\left\{j:\left(a_{j}, b_{j}\right) \neq(0,0)\right\} .
$$

(See [6],[5] for details).

An $\left(n, k_{1}, k_{2}\right)$ CSS code is defined by two classical binary codes $C_{1}$ and $C_{2}$ with the property that $C_{1} \subseteq C_{2}$. The code $C_{1}$ is an $\left[n, k_{1}\right]$ code and code $C_{2}$ is an $\left[n, k_{2}\right]$ code. By $C_{1}^{\perp}$ and $C_{2}^{\perp}$ we denote the Euclidean dual codes of $C_{1}$ and $C_{2}$ respectively. The CSS is a stabilizer code. The generator matrix of its associated code $C^{\perp}$ (in the binary form) is defined by

$$
G_{C^{\perp}}=\left[\begin{array}{ll}
G_{C_{1}} & 0 \\
0 & G_{C_{2}^{\perp}}
\end{array}\right]
$$

where $G_{C_{1}}$ and $G_{C_{2}^{\perp}}$ are generator matrices of $C_{1}$ and $C_{2}^{\perp}$ respectively.

We will say that a CSS code is balanced if $k_{1}=n-k_{2}$.

Define the ensemble of CSS codes as

$$
\mathcal{Q}_{C S S}=\left\{Q: Q \text { is an }\left(n, k_{1}, k_{2}\right) \text { CSS code }\right\} .
$$

We need the following lemmas for deriving the average enumerators of $\mathcal{Q}_{C S S}$. The proofs of the Lemmas are in the Appendix.

Remind that the binary Gaussian binomial coefficients are defined by

$$
\begin{gathered}
{\left[\begin{array}{l}
n \\
0
\end{array}\right]=1} \\
{\left[\begin{array}{c}
n \\
k
\end{array}\right]=\frac{\left(2^{n}-1\right)\left(2^{n-1}-1\right) \ldots\left(2^{n-k+1}-1\right)}{\left(2^{k}-1\right)\left(2^{k-1}-1\right) \ldots(2-1)},}
\end{gathered}
$$

and that

$$
\left[\begin{array}{c}
n \\
n-k
\end{array}\right]=\left[\begin{array}{l}
n \\
k
\end{array}\right] \text {. }
$$

Lemma 8: Let $\mathbf{v}, \mathbf{w} \in \mathbb{F}_{2}^{n} \backslash \mathbf{0}$ and $\mathbf{v} \cdot \mathbf{w}=0$. If $\mathbf{v} \in C_{1}$ and $\mathbf{w} \in C_{2}^{\perp}$ then code $C_{2}$ can be chosen in

$$
\left[\begin{array}{c}
n-2 \\
k_{2}-1
\end{array}\right]
$$

ways.

Lemma 9: The number of $\left[n, k_{2}\right]$ codes that contain a given $\left[n, k_{1}\right]$ code is equal to

$$
\left[\begin{array}{c}
n-k_{1} \\
k_{2}-k_{1}
\end{array}\right]
$$

Lemma 10: The number of $\left[n, k_{1}\right]$ codes in a given $\left[n, k_{2}\right]$ code is equal to

$$
\left[\begin{array}{l}
k_{2} \\
k_{1}
\end{array}\right] .
$$

Lemma 11: Let $\mathbf{v} \in \mathbb{F}_{2}^{n} \backslash \mathbf{0}$. The number of $\left[n, k_{1}\right]$ codes $C_{1}$ in a given $\left[n, k_{2}\right]$ code such that $\mathbf{v} \in C_{1}$ is equal to

$$
\left[\begin{array}{l}
k_{2}-1 \\
k_{1}-1
\end{array}\right] \text {. }
$$

Lemma 12: Let $C_{2}$ be an $\left[n, k_{2}\right]$ code and let $\mathbf{w} \in C_{2}^{\perp}$. Then $C_{2}$ can be constructed in

$$
\left[\begin{array}{c}
n-1 \\
k_{2}
\end{array}\right]
$$

ways.

Using the above Lemmas we will prove the following theorems that define the average distance distribution of $\left(n, k_{1}, k_{2}\right)$ CSS codes.

Theorem 13: Let $\mathbf{v}=(\mathbf{a}, \mathbf{0}) \in \mathbb{F}_{2}^{2 n}$, where $\mathbf{0}=$ $(0, \ldots, 0) \in \mathbb{F}_{2}^{n}$, and $\mathbf{a} \in \mathbb{F}_{2}^{n} \backslash \mathbf{0}$. Then $\mathbf{v}$ is contained in

$$
\left[\begin{array}{c}
n-1 \\
k_{1}-1
\end{array}\right]\left[\begin{array}{c}
n-k_{1} \\
k_{2}-k_{1}
\end{array}\right]
$$

codes with generators matrices of the form (29). The number of such vectors is $2^{n}-1$.

Proof: The number of $C_{1}$ codes that contain the vector $\mathbf{a}$ is

$$
\left[\begin{array}{c}
n-1 \\
k_{1}-1
\end{array}\right] \text {. }
$$

The number of $C_{2}$ codes that contain a given $C_{1}$ is defined in Lemma 9 This finishes the proof.

Theorem 14: Let $\mathbf{v}=(\mathbf{0}, \mathbf{a}) \in \mathbb{F}_{2}^{2 n}$, where $\mathbf{0}=$ $(0, \ldots, 0) \in \mathbb{F}_{2}^{n}$, and $\mathbf{a} \in \mathbb{F}_{2}^{n} \backslash \mathbf{0}$. Then $\mathbf{v}$ is contained in

$$
\left[\begin{array}{c}
n-1 \\
k_{2}
\end{array}\right]\left[\begin{array}{l}
k_{2} \\
k_{1}
\end{array}\right]
$$

codes with generators matrices of the form (29). The number of such vectors is $2^{n}-1$.

Proof: The number of codes $C_{2}$ such that $\mathbf{a} \in C_{2}^{\perp}$ is defined by Lemma12. The number of $C_{1}$ codes in a given $C_{2}$ is determined by Lemma 10

Theorem 15: Let $\mathbf{a}, \mathbf{b} \in \mathbb{F}_{2}^{n} \backslash \mathbf{0}$ and let $\mathbf{v}=(\mathbf{a}, \mathbf{b}) \in \mathbb{F}_{2}^{2 n}$ be a vector such that $\left|\left\{a_{j}=b_{j}=1\right\}\right|$ is a positive even number. Then $\mathbf{v}$ is contained in

$$
\left[\begin{array}{c}
n-2 \\
k_{2}-1
\end{array}\right]\left[\begin{array}{c}
k_{2}-1 \\
k_{1}-1
\end{array}\right]
$$

codes with generators matrices of the form (29). The number of such vectors is

$$
\sum_{i>0, i \text { is even }}^{n}\left(\begin{array}{l}
n \\
i
\end{array}\right) 3^{n-i} .
$$

Proof: Since $\left|\left\{a_{j}=b_{j}=1\right\}\right|$ is even we have $\mathbf{a} \cdot \mathbf{b}=0$. Hence we can construct CSS codes such that $\mathbf{a} \in C_{1}$ and $\mathbf{b} \in C_{2}^{\perp}$. The number of $C_{2}^{\perp}$ codes of this type, and therefore the number of $C_{2}$ codes, is defined by Lemma 8 . The number of $C_{1}$ codes with $\mathbf{a} \in C_{1}$ that are contained in a given $C_{2}$ is defined by Lemma 11 . 
Theorem 16: Let $\mathbf{a}, \mathbf{b} \in \mathbb{F}_{2}^{n} \backslash \mathbf{0}$ and let $\mathbf{v}=(\mathbf{a}, \mathbf{b}) \in \mathbb{F}_{2}^{2 n}$ be a vector such that $\left|\left\{a_{j}=b_{j}=1\right\}\right|$ is an odd number. Then $\mathbf{v}$ does not belong to any code with generator matrix of the form (29).

Proof: If $\mathbf{v}=(\mathbf{a}, \mathbf{b})$, with nonzero $\mathbf{a}$ and $\mathbf{b}$, then $\mathbf{a} \in C_{1}$ and $\mathbf{b} \in C_{2}^{\perp} \subseteq C_{1}^{\perp}$. At the same time $\left|\left\{a_{j}=b_{j}=1\right\}\right|$ is odd and therefore $\mathbf{a} \cdot \mathbf{b}=1$. A contradiction.

Theorem 17: Let $\mathbf{a}, \mathbf{b} \in \mathbb{F}_{2}^{n} \backslash \mathbf{0}$ and there is no $j$ with $a_{j}=1, b_{j}=1$. Then the vector $(\mathbf{a}, \mathbf{b})$ is contained in

$$
\left[\begin{array}{c}
n-2 \\
k_{2}-1
\end{array}\right]\left[\begin{array}{l}
k_{2}-1 \\
k_{1}-1
\end{array}\right]
$$

codes with generators matrices of the form 29. The number of such vectors is

$$
3^{n}-2\left(2^{n}-1\right)
$$

Proof: Since there is no $j$ such that $a_{j}=1$ and $b_{j}=1$ we have $\mathbf{a} \cdot \mathbf{b}=0$. Hence we can construct CSS codes such that $\mathbf{a} \in C_{1}$ and $\mathbf{b} \in C_{2}^{\perp}$. The number of such CSS codes is defined by Lemmas 8 and 11 .

Theorem 18: The average quantum enumerators of the ensemble $\mathcal{Q}_{C S S}$ is

$$
\begin{gathered}
\bar{B}_{0}^{\perp}=1 \\
\bar{B}_{j}^{\perp}=\frac{1}{c_{1}}\left[\left(\begin{array}{c}
n \\
j
\end{array}\right)\left(c_{2}-\frac{3}{2} c_{3}\right)+\frac{c_{3}}{2}\left(\begin{array}{l}
n \\
j
\end{array}\right) 3^{j}\right], j \geqslant 1 .
\end{gathered}
$$

and

$$
\begin{gathered}
\bar{B}_{0}=1, \\
\bar{B}_{j}=\frac{1}{2^{k_{1}+n-k_{2}} c_{1}} \\
\cdot\left(\left(\begin{array}{c}
n \\
j
\end{array}\right)\left[2^{n}\left(c_{2}-\frac{3}{2} c_{3}\right)+3^{j}\left(c_{1}-c_{2}+c_{3}\right)\right]\right), j \geqslant 1,
\end{gathered}
$$

where

$$
\begin{aligned}
c_{1} & =\left[\begin{array}{c}
n \\
k_{2}
\end{array}\right]\left[\begin{array}{l}
k_{2} \\
k_{1}
\end{array}\right], \\
c_{2} & =\left[\begin{array}{c}
n-1 \\
k_{1}-1
\end{array}\right]\left[\begin{array}{c}
n-k_{1} \\
k_{2}-k_{1}
\end{array}\right]+\left[\begin{array}{c}
n-1 \\
k_{2}
\end{array}\right]\left[\begin{array}{l}
k_{2} \\
k_{1}
\end{array}\right], \\
c_{3} & =\left[\begin{array}{c}
n-2 \\
k_{2}-1
\end{array}\right]\left[\begin{array}{l}
k_{2}-1 \\
k_{1}-1
\end{array}\right] .
\end{aligned}
$$

Proof: Remind that the weight of a vector $\mathbf{a}+\omega \mathbf{b}$ can be found according to 28).

It is easy to see that the number of vectors from Theorem 13 of weight $j$ is $\left(\begin{array}{l}n \\ j\end{array}\right)$. The same is true for vectors from Theorem 14. In the case of Theorem 15 we have that the number of vectors of weight $j$ is

$$
\sum_{i>0, i \text { is even }}^{j}\left(\begin{array}{l}
n \\
i
\end{array}\right)\left(\begin{array}{c}
n-i \\
j-i
\end{array}\right) 2^{j-i}
$$

and for Theorem 17 this number is

$$
\left(\begin{array}{l}
n \\
j
\end{array}\right)\left(2^{j}-2\right)
$$

Combining these results we obtain

$$
\begin{aligned}
\bar{B}_{j}^{\perp} & =\frac{1}{c_{1}}\left[\left(\begin{array}{c}
n \\
j
\end{array}\right) c_{2}\right. \\
& \left.+\left(\sum_{i>0, i \text { is even }}^{j}\left(\begin{array}{c}
n \\
i
\end{array}\right)\left(\begin{array}{c}
n-i \\
j-i
\end{array}\right) 2^{j-i}+\left(\begin{array}{c}
n \\
j
\end{array}\right)\left(2^{j}-2\right)\right) c_{3}\right] .
\end{aligned}
$$

Using the identities

$$
\left(\begin{array}{l}
n \\
i
\end{array}\right)\left(\begin{array}{c}
n-i \\
j-i
\end{array}\right)=\left(\begin{array}{l}
n \\
j
\end{array}\right)\left(\begin{array}{l}
j \\
i
\end{array}\right)
$$

and

$$
\sum_{i=0, i \text { is even }}^{j}\left(\begin{array}{l}
j \\
i
\end{array}\right) 2^{-i}=\frac{1}{2}\left[\left(1+\frac{1}{2}\right)^{j}+\left(1-\frac{1}{2}\right)^{j}\right],
$$

after simple manipulations, we obtain (30).

The generating function of the $q$-ary Krawtchouk polynomials (see [11, Ch.5.7]) is

$$
(1+(q-1) z)^{n-j}(1-z)^{j}=\sum_{r=0}^{n} \mathcal{K}_{r}(j) z^{r} .
$$

Hence, in the case $q=4$, the sum

$$
\sum_{j=0}^{n}\left(\begin{array}{l}
n \\
j
\end{array}\right) \mathcal{K}_{r}(j)
$$

is equal to the coefficient of $z^{r}$ of the polynomial

$$
\sum_{j=0}^{n}\left(\begin{array}{l}
n \\
j
\end{array}\right)(1+3 z)^{n-j}(1-z)^{j}=2^{n}(1+z)^{n} .
$$

Thus for $q=4$ we have

$$
\sum_{j=0}^{n}\left(\begin{array}{l}
n \\
j
\end{array}\right) \mathcal{K}_{r}(j)=2^{n}\left(\begin{array}{l}
n \\
r
\end{array}\right) .
$$

In a similar way it is easy to show that

$$
\sum_{j=0}^{n}\left(\begin{array}{l}
n \\
j
\end{array}\right) 3^{j} \mathcal{K}_{r}(j)=4^{n} \delta_{0, r} .
$$

Using these expressions together with the MacWilliams identities (6) we obtain (31).

For a set

$$
I \subseteq\left\{\bar{B}_{j}(Q)-\bar{B}_{j}^{\perp}(Q)<1\right\}
$$

we define the expurgated ensemble

$$
\mathcal{Q}_{C S S, e x}=\left\{Q \in \mathcal{Q}_{C S S}: B_{j}(Q)-B_{j}^{\perp}(Q)=0 \text { for all } j \in I\right\} \text {. }
$$

The average quantum enumerators of $\mathcal{Q}_{C S S, \text { ex }}$ can be upper bounded with the help of Theorems 6 and 18

Using Theorem 18 and the union bound we obtain a GilbertVarshamov type bound for finite length CSS codes.

Theorem 19: Let $d_{G V}\left(n, k_{1}, k_{2}\right)$ be the largest integer such that

$$
1-\sum_{j=1}^{d_{G V}\left(n, k_{1}, k_{2}\right)-1}\left(\bar{B}_{j}-\bar{B}_{j}^{\perp}\right)>0 .
$$

Then there is exists an $\left(n, k_{1}, k_{2}\right)$ CSS code with minimum distance $d_{G V}\left(n, k_{1}, k_{2}\right)$. 
In Fig 4 the quantity $1-\bar{F}$ for ensembles of $\mathcal{Q}_{s t a b}, \mathcal{Q}_{s t a b, e x}$, and $\mathcal{Q}_{C S S, \text { ex }}$ is presented. All considered codes have the same length $n=100$ and the same code rate $R=1 / 2$. One can see that codes from $\mathcal{Q}_{s t a b, e x}$ start to significantly outperform codes from $\mathcal{Q}_{\text {stab }}$ only at $p<10^{-3}$. For larger values of $p$ the expurgation does not play any role. This result can also be interpreted as that for $p>10^{-3}$ the minimum distance of a typical $[[50,22]]$ code does effect its fidelity. Another observation is that stabilizer codes are significantly better than CSS codes. Among CSS codes the balanced code $\left(k_{1}=n-k_{2}\right)$ happened to have significantly better performance than codes with $k_{1}<<n-k_{2}$ or $n-k_{2}<<k_{1}$.

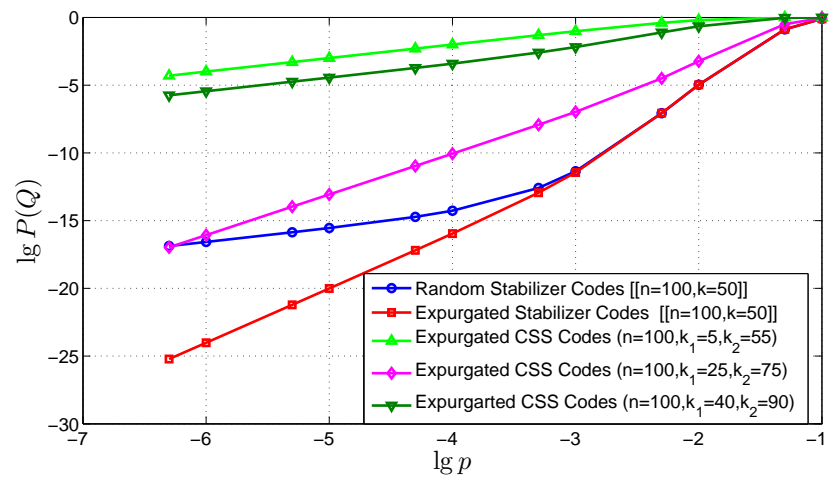

Fig. 4. Lower bounds on $1-\bar{F}$ for the ensemble of stabilizer codes and different ensembles of CSS codes; all codes have the same length $n=100$ and code rate $R=1 / 2$.

The obtained bounds allow us to estimate how the code performance changes with the code length. In Fig 5 we consider the case of the symmetric depolarizing channel with $p=0.01$ and codes of length $n \in[30,3000]$. We choose the target fidelity as $1-F_{\text {target }}=10^{-4}$. For a given code of length $n$ we find the largest code rate $R$ such that $1-\bar{F} \leqslant 1-F_{\text {target }}$. One can see that in the case of the ensemble $\mathcal{Q}_{s t a b}$ the code rate is approaching the capacity lower bound $\underline{C}(p)=1-2 H(p)$ $(H(p)$ is defined below in (32)) as the code length grows. At the same time for small code lengths, like $n=50$ or 100 , the code rate should be significantly lower than $\underline{C}(p)$ in order to have $1-\bar{F} \leqslant 1-F_{\text {target }}$

Fig 5 also shows that CSS codes have a significant data rate loss compared to stabilizer codes of the same length. It is unclear, however, whether this loss disappears in the asymptotic regime as the code length tends to infinity. We will answer this question in the next Section.

\section{RELIABILITy FunCtions ANd CAPACITy LOWER BOUNDS}

In this Section we are interested in the regime when the code length $n$ tends to infinity and the code rate stays constant.

We start with studying asymptotic behavior of the average enumerators $\hat{B}_{j}$ and $\hat{B}_{j}^{\perp}$ for expurgated ensembles of stabilizer and CSS codes.

Through the rest of the paper

$$
\begin{aligned}
T(x, y) & =x \log _{4}(3)-x \log _{4}(y)-(1-x) \log _{4}(1-y), \\
H(x) & =T(x, x) .
\end{aligned}
$$

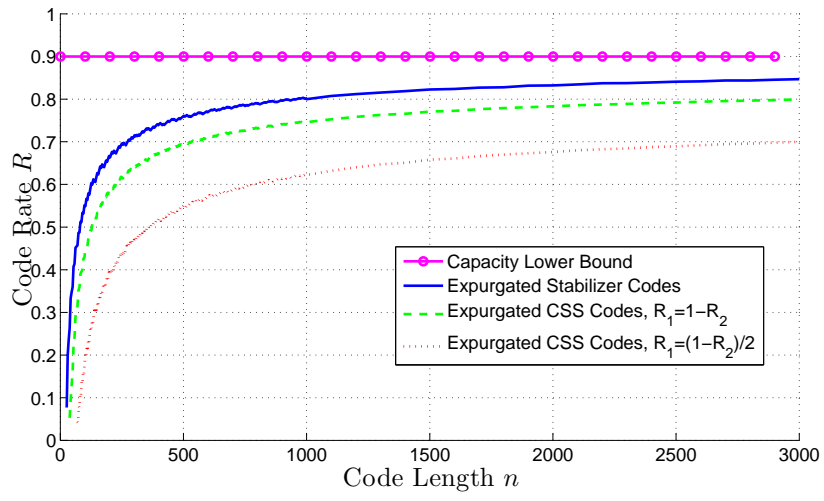

Fig. 5. Code Rate as the function of code length in the case of symmetric depolarizing channel with $p=0.01$ and $1-\bar{F} \leqslant 10^{-4}$

\section{A. Stabilizer and Linear Stabilizer Codes}

Let $\mathcal{Q}_{s t a b}$ be the ensemble of all $[[n, k]]$ stabilizer codes. From (21) and (22) we obtain

$$
\begin{aligned}
& \bar{b}_{\omega}^{\perp}=\frac{1}{n} \log _{4} \bar{B}_{\lfloor\omega n\rfloor}^{\perp}=H(\omega)-\frac{R+1}{2}+o(1), \\
& \bar{b}_{\omega}=\frac{1}{n} \log _{4} \bar{B}_{\lfloor\omega n\rfloor}=H(\omega)+\frac{R-1}{2}+o(1) .
\end{aligned}
$$

Theorem 6 allows obtaining expurgated ensembles that give good fidelity bounds for finite length quantum codes. In the asymptotic case it is more convenient to form expurgated ensemble in the way proposed in [1]. For a positive $\rho>0$ we define the expurgated ensemble as

$$
\begin{aligned}
& \mathcal{Q}_{s t a b, E X} \\
= & \left\{Q \in \mathcal{Q}_{s t a b}: B_{j}(Q)-B_{j}^{\perp}(Q) \leqslant n^{1+\rho}\left(\bar{B}_{j}-\bar{B}_{j}^{\perp}\right) \text { for all } j\right\} .
\end{aligned}
$$

From Markov's inequality and union bound it follows that for randomly chosen $Q \in \mathcal{Q}_{\text {stab }}$ we have

$$
\begin{aligned}
& \operatorname{Pr}\left(B_{j}(Q)-B_{j}^{\perp}(Q) \leqslant n^{1+\rho}\left(\bar{B}_{j}-\bar{B}_{j}^{\perp}\right) \text { for all } j\right) \\
\geqslant & 1-\frac{1}{n^{\rho}}, \rho>0 .
\end{aligned}
$$

Hence for growing $n$ we have that almost any code from $\mathcal{Q}_{s t a b}$ belongs to $\mathcal{Q}_{s t a b, E X}$.

Remark Note that the ensemble $\mathcal{Q}_{s t a b, E X}$ is different from the expurgated ensemble $\mathcal{Q}_{\text {stab,ex }}$ defined in Section IV While the polynomial factor $n^{\rho}$ does not affect asymptotic results (as it will be shown later in this Section), it does not allow achieving good bounds on $1-\bar{F}$ for finite length codes. In fact, for finite length codes bounds derived from $\mathcal{Q}_{s t a b, E X}$ are worse than similar bounds derived from the unexpurgated ensemble $\mathcal{Q}_{\text {stab }}$.

Denote

$$
\delta_{G V}(R)=\lim _{n \rightarrow \infty} \frac{d_{G V}(n, R n)}{n}=H^{-1}\left(\frac{1-R}{2}\right),
$$

where $d_{G V}(n, R n)$ is defined in Theorem 5

From (33) and (34) it follows that for sufficiently large $n$ we have

$$
\frac{1}{n} \log _{4} n^{1+\rho}\left(\bar{B}_{\lfloor\omega n\rfloor}-\bar{B}_{\lfloor\omega n\rfloor}^{\perp}\right)<1, \text { if } \omega<H^{-1}\left(\frac{1-R}{2}\right) .
$$


Taking into account that $B_{j}(Q)-B_{j}(Q)^{\perp}$ are integers, we obtain that for any $Q \in \mathcal{Q}_{s t a b, E X}$ and sufficiently large $n$ :

$$
B_{j}(Q)-B_{j}(Q)^{\perp}=0,1 \leqslant j \leqslant\left(\delta_{G V}(R)-\epsilon\right) n, \epsilon>0 .
$$

For $\omega \geqslant \delta_{G V}(R)$ we have

$$
\begin{aligned}
\tilde{b}_{\omega} & =\frac{1}{n} \log _{4}\left(B_{\lfloor\omega n\rfloor}(Q)-B_{\lfloor\omega n\rfloor}^{\perp}(Q)\right) \\
& \leqslant \frac{1}{n} \log _{4} n^{1+\rho}\left(\bar{B}_{\lfloor\omega n\rfloor}-\bar{B}_{\lfloor\omega n\rfloor}^{\perp}\right) \\
& =\bar{b}_{\omega}+o(1) \\
& =H(\omega)+\frac{R-1}{2}+o(1), \omega \geqslant \delta_{G V}(R) .
\end{aligned}
$$

The ensemble of $\mathcal{Q}_{\text {lin.stab }}$ can be analyzed in a very similar way. Starting from the expressions (17), and (18), and further defining the ensemble $\mathcal{Q}_{\text {lin.stab,EX }}$ in the same way as in (35), we obtain that equations (37) and (38) also hold for $\mathcal{Q}_{\text {lin.stab }, E X}$.

\section{B. CSS codes}

Now we consider the ensemble $\mathcal{Q}_{C S S}$ of all $\left(n, k_{1}, k_{2}\right)$ CSS codes with the average enumerators $\bar{B}_{j}^{\perp}$ and $\bar{B}_{j}$.

Define

$$
[n]=\left(2^{n}-1\right)\left(2^{n-1}-1\right) \cdot \ldots \cdot(2-1) .
$$

The following identities are well known (see [11, Ch.15.2])

$$
\begin{gathered}
{\left[\begin{array}{c}
n \\
k
\end{array}\right]=\frac{[n]}{[k][n-k]},} \\
{\left[\begin{array}{c}
n \\
k_{2}
\end{array}\right]\left[\begin{array}{c}
k_{2} \\
k_{1}
\end{array}\right]=\left[\begin{array}{c}
n-k_{1} \\
k_{2}-k_{1}
\end{array}\right]\left[\begin{array}{c}
n \\
k_{1}
\end{array}\right] .}
\end{gathered}
$$

We use these identities in order of studying the asymptotic behavior of the ratios $c_{2} / c_{1}$ and $c_{3} / c_{1}$ for $c_{1}, c_{2}$, and $c_{3}$ defined in Theorem 18. We start with the first term of $c_{2}$ and get

$$
\begin{aligned}
& \frac{\left[\begin{array}{c}
n-1 \\
k_{1}-1
\end{array}\right]\left[\begin{array}{c}
n-k_{1} \\
k_{2}-k_{1}
\end{array}\right]}{c_{1}}=\frac{\left[\begin{array}{c}
n-1 \\
k_{1}-1
\end{array}\right]}{\left[\begin{array}{c}
n \\
k_{1}
\end{array}\right]}=\frac{[n-1]\left[k_{1}\right]}{[n]\left[k_{1}-1\right]} \\
= & \frac{2^{k_{1}}-1}{2^{n}-1} .
\end{aligned}
$$

Similarly for the second term of $c_{2}$ we have

$$
\frac{\left[\begin{array}{c}
n-1 \\
k_{2}
\end{array}\right]\left[\begin{array}{l}
k_{2} \\
k_{1}
\end{array}\right]}{c_{1}}=\frac{\left[\begin{array}{c}
n-1 \\
k_{2}
\end{array}\right]}{\left[\begin{array}{c}
n \\
k_{2}
\end{array}\right]}=\frac{2^{n-k_{2}}-1}{2^{n}-1} .
$$

Hence we have

$$
\frac{1}{n} \log _{2}\left(\frac{c_{2}}{c_{1}}\right)=\max \left\{\frac{k_{1}}{n}-1,-\frac{k_{2}}{n}\right\}+o(1) .
$$

Next

$$
\begin{aligned}
\frac{c_{3}}{c_{1}}= & \frac{[n-2]}{\left[k_{2}-1\right]\left[n-k_{2}-1\right]} \frac{\left[k_{2}\right]\left[n-k_{2}\right]}{[n]} \\
& \frac{\left[k_{2}-1\right]}{\left[k_{1}-1\right]\left[k_{2}-k_{1}\right]} \frac{\left[k_{1}\right]\left[k_{2}-k_{1}\right]}{\left[k_{2}\right]} \\
= & \frac{\left(2^{k_{2}}-1\right)\left(2^{n-k_{2}}-1\right)\left(2^{k_{1}}-1\right)}{\left(2^{n}-1\right)\left(2^{n-1}-1\right)\left(2^{k_{2}}-1\right)} .
\end{aligned}
$$

From this we have

$$
\frac{1}{n} \log _{2}\left(\frac{c_{3}}{c_{1}}\right)=\frac{k_{1}}{n}-1-\frac{k_{2}}{n}+o(1) .
$$

Now, denoting by

$$
R_{1}=\log _{2} \frac{\left|C_{1}\right|}{n} \text { and } R_{2}=\log _{2} \frac{\left|C_{2}\right|}{n}
$$

the codes rates of $C_{1}$ and $C_{2}$ respectively, we obtain the following Theorem.

Theorem 20:

$$
\begin{aligned}
& \bar{b}_{\xi}^{\perp}=\frac{1}{n} \log _{4} \bar{B}_{\lfloor\xi n\rfloor}^{\perp} \\
= & H(\xi)-\xi \log _{4}(3) \\
+ & \max \left\{\frac{R_{1}-1}{2},-\frac{R_{2}}{2}, \xi \log _{4}(3)+\frac{R_{1}-1-R_{2}}{2}\right\}+o(1), \\
& \bar{b}_{\xi}=\frac{1}{n} \log _{4} \bar{B}_{\lfloor\xi n\rfloor} \\
= & H(\xi)-\xi \log _{4}(3) \\
+ & \max \left\{\frac{R_{2}-1}{2},-\frac{R_{1}}{2}, \xi \log _{4}(3)+\frac{R_{2}-1-R_{1}}{2}\right\}+o(1) .
\end{aligned}
$$

For a positive $\rho>0$, we define the expurgated ensemble of CSS codes as

$$
\begin{aligned}
\mathcal{Q}_{C S S, E X}= & \left\{Q \in \mathcal{Q}_{C S S}:\right. \\
& \left.B_{j}(Q)-B_{j}^{\perp}(Q) \leqslant n^{1+\rho}\left(\bar{B}_{j}-\bar{B}_{j}^{\perp}\right)\right\} .
\end{aligned}
$$

Remark Again we note that the ensemble $\mathcal{Q}_{C S S, E X}$ is different from $\mathcal{Q}_{C S S, e x}$ defined in Section $\mathbf{V}$ The ensemble $\mathcal{Q}_{C S S, E X}$ is convenient for asymptotic analysis, but gives bad results for finite length CSS codes.

Theorem 20 allows us to find the asymptotic expression for $d_{G V, C S S}\left(n, k_{1}, k_{2}\right)$ from Theorem 19. Let

$$
\delta_{G V, C S S}\left(R_{1}, R_{2}\right)=\lim _{n \rightarrow \infty} \frac{d_{G V, C S S}\left(n, R_{1} n, R_{2} n\right)}{n} .
$$

Taking into account that $B(Q)-B(Q)^{\perp}$ are integers and that $\bar{b}_{\xi}>\bar{b}_{\xi}^{\perp}, \xi \in(0,1)$, we conclude that $\delta\left(R_{1}, R_{2}\right)$ is the root of the equation $\bar{b}_{\xi}=0$. After simple calculations we obtain

$$
\delta_{G V, C S S}\left(R_{1}, R_{2}\right)=H_{2}^{-1}\left(\min \left(R_{1}, 1-R_{2}\right)\right),
$$

where

$$
H_{2}(x)=-x \log _{2}(x)-(1-x) \log _{2}(1-x)
$$

is the binary entropy. Note that if $R_{1}=1-R_{2}$ then $R_{1}=$ $(1-R) / 2$ we get the usual Gilbert-Varshamov bound for CSS codes [12, Ch.10.4.2]:

$$
\delta_{G V, C S S}(R)=H_{2}^{-1}\left(\frac{1-R}{2}\right) .
$$

Summarizing this, we obtain the following result.

Theorem 21: Let $Q \in \mathcal{Q}_{C S S, E X}$. Then

1) for any $\epsilon>0$ and sufficiently large $n$ we have

$$
B_{j}(Q)-B_{j}^{\perp}(Q)=0,1 \leqslant j \leqslant\left(\delta_{C S S, G V}\left(R_{1}, R_{2}\right)-\epsilon\right) n,
$$


2) for $\omega>\delta_{C S S, G V}\left(R_{1}, R_{2}\right)$ we have

$$
\tilde{b}_{\omega}=\frac{1}{n} \log _{4}\left(B_{\lfloor\omega n\rfloor}(Q)-B_{\lfloor\omega n\rfloor}^{\perp}(Q)\right) \leqslant \bar{b}_{\omega}+o(1),
$$

where $\bar{b}_{\omega}$ is defined in Theorem 20 .

Examples of average quantum enumerators for stabilizer and CSS codes are shown in Fig 6 and Fig 7 The figures show that at certain range of $\omega$ quantum enumerators of CSS codes are exponentially larger than their stabilizer counterparts. We see in the next subsection that this leads to an exponential loss of performance of CSS codes.

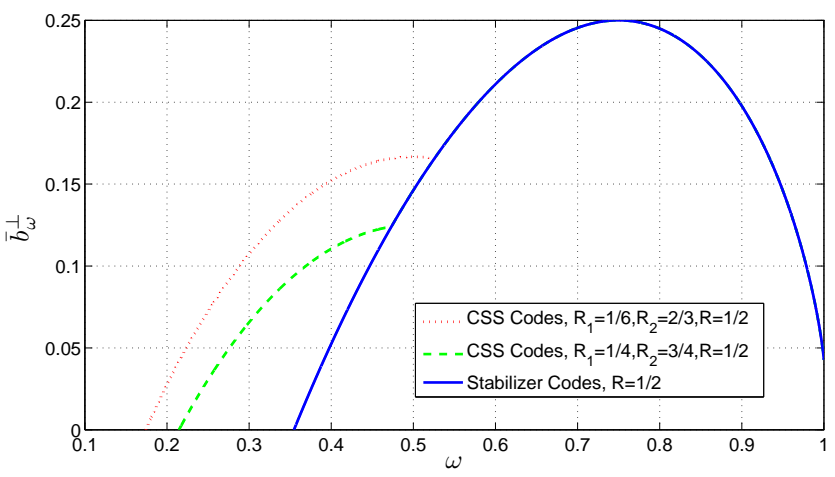

Fig. 6. Average Quantum Enumerators $\bar{b}_{\omega}^{\perp}$ of Stabilizer and CSS codes (only positive part of $\bar{b}_{\omega}^{\perp}$ is shown)

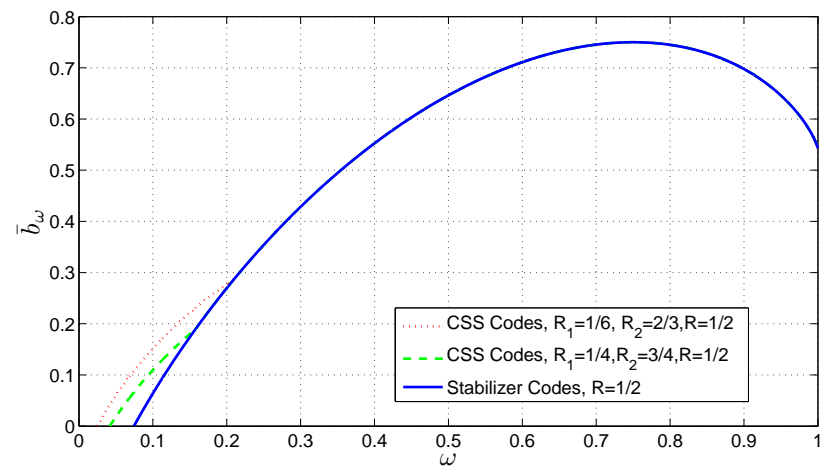

Fig. 7. Average Quantum Enumerators $\bar{b}_{\omega}$ of Stabilizer and CSS codes (only positive part of $\bar{b}_{\omega}$ is shown)

\section{Reliability Functions}

Let $\mathcal{Q}$ be an ensemble of quantum codes of rate $R$ and length $n$. For the quantum depolarizing channel with the channel probability $p$ define

$$
E(n, R, p)=\sup _{Q \in \mathcal{Q}}-\frac{1}{n} \log _{4}(1-F(Q))
$$

The reliability function, which is also called the error exponent, of $\mathcal{Q}$ is defined as

$$
E(R, p)=\lim \inf _{n \rightarrow \infty} E(n, R, p) .
$$

When $E(R, p)$ is positive it shows how fast $1-F$ approaches zero as $n \rightarrow \infty$. Thus for given $R$ and $p$ we want that $E(R, p)$ be as large as possible.

In what follows we will compute lower bounds on $E(R, p)$ for the ensembles of stabilizer and CSS codes. In order of doing this we analyze the exponent of (14) with the quantum enumerators of stabilizer and CSS codes.

We start with computing the exponent of $G(m, w)$ defined in (11). Let

$$
\mu=\frac{m}{n}, \omega=\frac{w}{n}, \eta=\frac{h}{n}, \text { and } \tau=\frac{t}{n} .
$$

For the $h$-th term of the second sum of $G(m, w)$ we have

$$
\begin{aligned}
& \frac{1}{n} \log _{4}\left(\begin{array}{c}
w-t \\
h
\end{array}\right)\left(\begin{array}{c}
n-w \\
m-t-h
\end{array}\right) 3^{m-t-h} \\
= & (\omega-\tau) H\left(\frac{\eta}{\omega-\tau}\right)-\eta \log _{4}(3) \\
+ & (1-\omega) H\left(\frac{\mu-\tau-\eta}{1-\omega}\right)+o(1) .
\end{aligned}
$$

The roots of the derivative of this expression with respect to $\eta$ are

$$
\eta_{1}=a+\frac{1}{4} \sqrt{b}, \eta_{2}=a-\frac{1}{4} \sqrt{b}
$$

where

$$
a=\frac{\omega}{2}-\frac{\tau}{4}+\frac{\mu}{2}-\frac{3}{4}
$$

and

$$
\begin{aligned}
b & =4 \omega^{2}-12 \omega \tau+16 \omega \mu-12 \omega \\
& +9 \tau^{2}-12 \tau \mu+6 \tau+4 \mu^{2}-12 \mu+9 .
\end{aligned}
$$

It is not difficult to show that the maximum of (45) is achieved at $\eta_{1}$ (in fact $\eta_{2}$ is always negative when $\mu$, $\omega$, and $\tau$ belong to their summation ranges). Further one can show that $\eta_{1}<$ $(\omega-\tau) / 2$ when $\mu, \omega$, and $\tau$ belong to their summation ranges. Thus the maximum of (45) is achieved at

$$
\eta^{*}=(\omega-\tau) / 2
$$

Using $\eta^{*}$ we compute the exponent of the $t$-th term of the first sum of $G(m, w)$ and obtain

$$
\begin{aligned}
& \frac{1}{n} \log _{4}\left(\left(\begin{array}{c}
w \\
t
\end{array}\right) 2^{t}\left(\begin{array}{c}
w-t \\
\frac{w-t}{2}
\end{array}\right)\left(\begin{array}{c}
n-w \\
\frac{2 m-t-w}{2}
\end{array}\right) 3^{\frac{2 m-t-w}{2}}\right) \\
= & \omega H\left(\frac{\tau}{\omega}\right)-\tau \log _{4}(3)+\frac{\omega}{2} \\
& +(1-\omega) H\left(\frac{2 \mu-\tau-\omega}{2(1-\omega)}\right) .
\end{aligned}
$$

The derivative of this expression in $\tau$ has three roots. Two of them take either negative or complex values when $\mu$ and $\omega$ belong to their summation ranges. The root that takes nonnegative values is

$$
\tau^{*}=\frac{(a+36 \sqrt{b})^{1 / 3}}{12}-\frac{12 c}{\left((a+36 \sqrt{b})^{1 / 3}\right)}+\frac{1}{3} \omega+\frac{2}{3} \mu-\frac{1}{2},
$$


where

$$
\begin{aligned}
a= & 672 \mu \omega^{2}+192 \omega \mu^{2}-720 \omega \mu-80 \omega^{3}-396 \omega^{2} \\
& +432 \omega+512 \mu^{3}-1152 \mu^{2}+864 \mu-216 ; \\
b= & 24 \mu \omega^{3}+528 \mu^{2} \omega^{2}-147 \omega^{4}-144 \mu \omega^{2}+72 \omega^{3}-12 \omega^{6} \\
& +136 \omega^{5}+192 \omega^{4} \mu^{2}-112 \omega^{5} \mu-96 \omega^{3} \mu^{2}-48 \omega^{4} \mu \\
& -640 \omega^{2} \mu^{3}+256 \omega^{2} \mu^{4} ; \\
c= & -\frac{1}{9} \omega \mu-\frac{7}{36} \omega^{2}+\frac{1}{3} \omega-\frac{4}{9} \mu^{2}+\frac{2}{3} \mu-\frac{1}{4} .
\end{aligned}
$$

We omit large analytical expressions for other two roots.

Denote

$$
\begin{aligned}
f(\mu, \omega)= & \omega H\left(\frac{\tau}{\omega}\right)-\tau^{*} \log _{4}(3) \\
& +\frac{\omega}{2}+(1-\omega) H\left(\frac{2 \mu-\tau^{*}-\omega}{2(1-\omega)}\right) .
\end{aligned}
$$

Remark As we noted in the end of section VI-A the ensembles $\mathcal{Q}_{s t a b, E X}$ and $\mathcal{Q}_{\text {lin.stab,EX }}$ have the same $\tilde{b}_{\omega}$. From this it follows that these ensembles have the same reliability function. Therefore all results presented below for stabilizer codes are also valid for linear stabilizer codes.

For the ensembles $\mathcal{Q}_{s t a b, E X}$ and $\mathcal{Q}_{C S S, E X}$ defined in (35) and (41) respectively and for $\bar{N}(m)$ defined in (15) we have

$$
\frac{1}{n} \log _{4} N(\lfloor\mu n\rfloor)=\max _{\delta<\omega<2 \mu}\left\{\tilde{b}_{\omega}+f(\mu, \omega)\right\},
$$

and further, according to (14),

$$
\begin{aligned}
E(R, p) & \geqslant \underline{E}(R, p)=\max _{\delta / 2<\mu<1}\left\{\mu \log _{4}(p / 3)\right. \\
& +(1-\mu) \log _{4}(1-p) \\
& +\min \left\{H(\mu), \max _{\delta<\omega<2 \mu}\left\{\tilde{b}_{\omega}+f(\mu, \omega)\right\}\right\}
\end{aligned}
$$

where $\delta$ is either $\delta_{G V}(R)$ or $\delta_{G V, C S S}\left(R_{1}, R_{2}\right)$ and $\tilde{b}_{\omega}$ is defined either in (38) or (44) respectively.

Numerical computation of $\underline{E}(R, p)$, for both $\mathcal{Q}_{s t a b, E X}$ and $\mathcal{Q}_{C S S, E X}$, is easy. The function inside of $\max _{\delta<\omega<2 \mu}\{\cdot\}$ and the function inside of $\max _{\delta / 2<\mu<1}\{\cdot\}$ were found to be concave by inspection, for parameter values tested. In the case of $\mathcal{Q}_{\text {stab,EX }}$ numerical computations show that $\underline{E}(R, p)$ coincides with Gallager's exponent (reliability function) of random classical quaternary code of rate $(R+1) / 2$. It also coincides with the reliability function found in [9],[10],[3]:

$$
\begin{aligned}
& E_{\text {stab }}(R, p) \geqslant \underline{E}(R, p) \\
&= \begin{cases}-\delta_{G V}\left(\frac{R+1}{2}\right) \log _{4}\left(\sqrt{\frac{4}{3} p(1-p)}\right. & \\
\left.+\frac{2}{3} p\right), & 0 \leqslant R \leqslant R_{\text {min }} \\
1-\log _{4}(1+2 p+\sqrt{12 p(1-p)}) & \\
-\frac{R+1}{2}, & R_{\text {min }} \leqslant R \leqslant R_{c r} \\
T\left(\delta_{G V}\left(\frac{R+1}{2}\right), p\right)-1+\frac{R+1}{2}, & R_{c r} \leqslant R \leqslant \underline{C}(p)\end{cases}
\end{aligned}
$$

where

$$
\begin{aligned}
\underline{C}(p) & =1-2 H(p), \\
R_{c r} & =\max \left\{0,1-2 H\left(\frac{\sqrt{3 p}}{\sqrt{3 p}+\sqrt{1-p}}\right)\right\}, \\
R_{\text {min }} & =\max \left\{0,1-2 H\left(\frac{3 \alpha}{1+3 \alpha}\right)\right\},
\end{aligned}
$$

and

$$
\alpha=\sqrt{\frac{4}{3} p(1-p)}+\frac{2}{3} p .
$$

The reliability function for the depolarizing channel with $p=0.01$ are shown in Fig 8 . One can see that stabilizer codes slightly outperformed balanced CSS codes $\left(R_{1}=1-R_{2}\right)$, and that balanced CSS codes significantly outperform unbalanced CSS codes with $R_{1}=\left(1-R_{2}\right) / 2$.

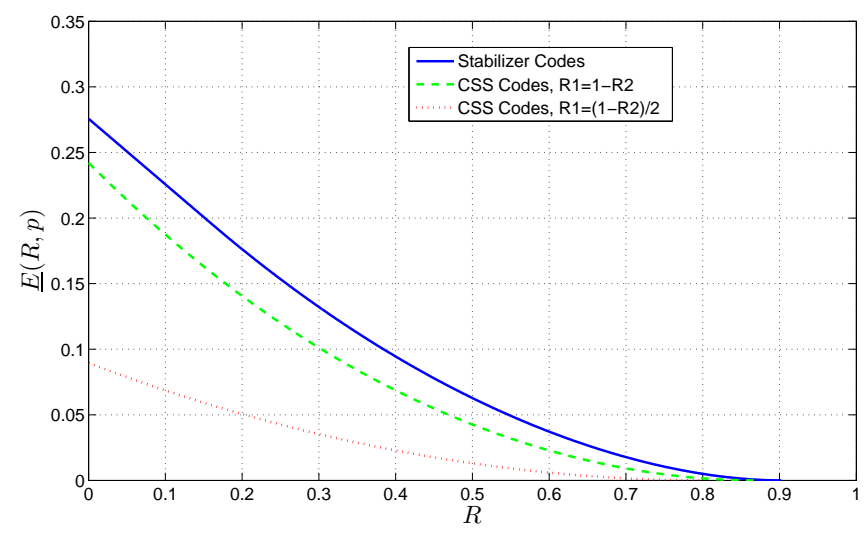

Fig. 8. Error Exponents for Random Stabilizer and CSS Codes in symmetric depolarizing channel with $p=0.01$

We can use the function $\underline{E}(R, p)$ for obtaining a lower bound $\underline{C}(p)$ on the capacity of an ensemble $\mathcal{Q}$ of quantum codes. For an ensemble $\mathcal{Q}$ of quantum codes and for given channel error $p$ we define $\underline{C}(p)$ by

$$
\underline{C}_{\mathcal{Q}}(p)=\max \{R: \underline{E}(R, p)>0\} .
$$

The capacity lower bounds for stabilizer, balanced and unbalanced CSS codes are shown in Fig 9 . We see again that stabilizer codes slightly outperform balanced CSS codes, and that CSS codes with $R_{1}=\left(1-R_{2}\right) / 2$ are significantly weaker than stabilizer and balanced CSS codes.

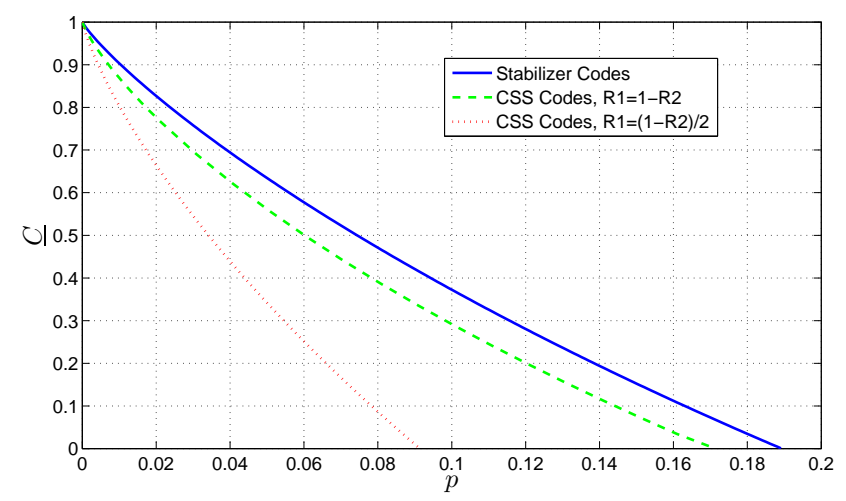

Fig. 9. Capacity Lower Bounds for Stabilizer and CSS Codes

\section{APPENDIX}

Proof: (Lemma 8) Any $C_{2}^{\perp}$ uniquely defines the code $C_{2}$. So it is enough to count in how many ways code $C_{2}^{\perp}$ can be 
constructed. Any vector from $C_{2}^{\perp}$ should be orthogonal to $\mathbf{v}$. There are $\left(2^{n-1}-1\right)$ nonzero vectors that are orthogonal to $\mathbf{v}$. We use $\mathbf{w}$ as the first basis vector of $C_{2}^{\perp}$. Other $n-k_{2}-1$ basis vectors can be chosen in

$$
\left(2^{n-1}-2\right)\left(2^{n-1}-2^{2}\right) \ldots\left(2^{n-1}-2^{n-k_{2}-1}\right)
$$

ways. In any such code its basis vectors can be chosen in

$$
\left(2^{n-k_{2}}-2\right)\left(2^{n-k_{2}}-2^{2}\right) \ldots\left(2^{n-k_{2}}-2^{n-k_{2}-1}\right)
$$

ways. The ratio of the above expressions is equal to

$$
\left[\begin{array}{c}
n-2 \\
n-k_{2}-1
\end{array}\right]=\left[\begin{array}{c}
n-2 \\
k_{2}-1
\end{array}\right]
$$

and it defines the number of ways in which code $C_{2}^{\perp}$ and code $C_{2}$ can be constructed.

Proof: (Lemma 9] Any $k_{1}$ basis vectors of the $\left[n, k_{1}\right]$ code can be chosen as the fist $k_{1}$ basis vectors of an $\left[n, k_{2}\right]$ code. Other $k_{2}-k_{1}$ vectors can be chosen in

$$
\left(2^{n}-2^{k_{1}}\right)\left(2^{n}-2^{k_{1}+1}\right) \ldots\left(2^{n}-2^{k_{2}-1}\right)
$$

ways. In any such $\left[n, k_{2}\right]$ code its $\left(k_{2}-k_{1}\right)$ basis vectors can be chosen in

$$
\left(2^{k_{2}}-2^{k_{1}}\right)\left(2^{k_{2}}-2^{k_{1}+1}\right) \ldots\left(2^{k_{2}}-2^{k_{2}-1}\right)
$$

ways. The ratio of the above expressions completes the proof.

Proof: (Lemma 10) The number of nonzero vectors in an $\left[n, k_{2}\right]$ code is $2^{k_{2}}-1$. Hence a basis of an $\left[n, k_{1}\right]$ code can be chosen in

$$
\left(2^{k_{2}}-1\right)\left(2^{k_{2}}-2\right) \ldots\left(2^{k_{2}}-2^{k_{1}-1}\right)
$$

ways. In any such $\left[n, k_{1}\right]$ code we can choose a basis in

$$
\left(2^{k_{1}}-1\right)\left(2^{k_{1}}-2\right) \ldots\left(2^{k_{1}}-2^{k_{1}-1}\right)
$$

ways. The number of distinct $\left[n, k_{1}\right]$ codes in a given $\left[n, k_{2}\right]$ code is equal to the ratio of the above expressions.

Proof: (Lemma 11). The proof is similar to the proof of Lemma 10

Proof: (Lemma 12) Nonzero vectors from $C_{2}$ must be orthogonal to $\mathbf{w}$. There are $2^{n-1}-1$ such vectors. Hence $k_{2}$ basis vectors of $C_{2}$ can be chosen in

$$
\left(2^{n-1}-1\right)\left(2^{n-1}-2\right) \cdot \ldots \cdot\left(2^{n-1}-2^{k_{2}-1}\right)
$$

ways. In a given $\left[n, k_{2}\right]$ code a basis can be chosen in

$$
\left(2^{k_{2}}-1\right)\left(2^{k_{2}}-2\right) \cdot \ldots \cdot\left(2^{k_{2}}-2^{k_{2}-1}\right)
$$

ways. The ratio of the above expressions competes the proof.

\section{ACKNOWLEDGEMENT}

This research was supported by the Intelligence Advanced Research Projects Activity (IARPA) via Department of Interior National Business Center contract number D11PC20165. The U.S. Government is authorized to reproduce and distribute reprints for Governmental purposes notwithstanding any copyright annotation thereon. The views and conclusions contained herein are those of the authors and should not be interpreted as necessarily representing the official policies or endorsements, either expressed or implied, of IARPA, DoI/NBC, or the U.S. Government.

\section{REFERENCES}

[1] A. Ashikhmin, A. Barg, E. Knill, S. Litsyn, "Quantum error detection .II. Bounds," IEEE Transactions on Information Theory, 46, 2000, pp.789800.

[2] A. Ashikhmin, E. Knill, "Nonbinary quantum stabilizer codes," IEEE Transactions on Information Theory, 47, 2001, pp.3065-3072.

[3] A. Barg, "A Lower-Rate Bound on the Reliability of a Quantum Discrete Memoryless Channel," IEEE Transactions on Information Theory, 48, 2002, pp.3096-3100.

[4] R. Blahut, "Theory and Practice of Error Control Codes," AddisonWesley Publishing Company, Inc., 1983.

[5] A. R. Calderbank, E. .M. Rains, P. M. Shor, N. J. A. Sloane, "Quantum error correction via codes over GF(4)," IEEE Transactions on Information Theory, 44, pp.1369-1387, 1998.

[6] A. R. Calderbank and P. W. Shor, Good quantum error correcting codes exist, Phys. Rev. A, 54, pp. 1098-1105, 1996.

[7] K. Feng and Z. Ma, "A finite Gilbert-Varshamov bound for pure stabilizer quantum codes," IEEE Trans. on Information Theory, 50, pp.3323-3325, 2004.

[8] D. Gottesman, "A class of quantum error-correcting codes saturating the quantum Hamming bound," Phys. Rev. A, 54, pp.1862-1868, 1996.

[9] M. Hamada, Exponential lower bound on the highest fidelity achievable by quantum error-correcting codes, Phys. Rev. A, 65, pp. 1-4, 2002. Paper no. 052305. E-Print, quant-ph/0109114 LANL, 2001.

[10] M. Hamada, "Lower bounds on the quantum capacity and highest error exponent of general memoryless channels," IEEE Trans. on Information Theory, 48, pp.2547 - 2557, 2002.

[11] F. J. MacWilliams and N. J. A. Sloane, "The Theory of Error Correcting Codes," North-Holland, 1977.

[12] M. A. Nielsen and I. .L. Chuang, "Quantum Computation and Quantum Information," Cambridge University Press, 2000.

[13] G. Poltyrev, "Bounds on the decoding error probability of binary linear codes via their spectra," IEEE Trans. on Information Theory, 40, pp.1284 - 1292, 1994.

[14] Y. Polyanskiy, H.V. Poor, S. Verdu, "Channel Coding Rate in the Finite Blocklength Regime," IEEE Trans. on Information Theory, 56, pp.2307 - 2359, 2010.

[15] J. Preskill, Lecture Notes for Physics 229: Quantum Information and Computation, Pasadena, CA:Calif. Inst. Technol., 1999.

[16] P. Shor, R. Laflamme, "Quantum analog of the MacWilliams identities in classical coding theory," Phys. Rev. Lett. 78, pp. 1600-1602, 1997.

[17] A. M. Steane, Multiple particle interference and error correction, Proc. the Royal Soc. of London A, 452, pp. 2551-2577, 1996.

[18] A. M. Steane, Error correcting codes in quantum theory, Phys. Rev. Lett., 77, pp.793-797, 1996. 